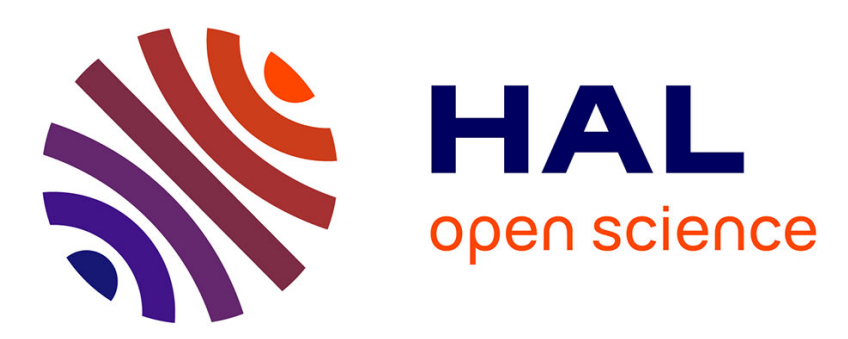

\title{
Dynamics of perceptual decisions about symmetry in visual cortex
}

Peter J. Kohler, Benoit R. Cottereau, Anthony M. Norcia

\section{To cite this version:}

Peter J. Kohler, Benoit R. Cottereau, Anthony M. Norcia. Dynamics of perceptual decisions about symmetry in visual cortex. NeuroImage, 2018, 167, pp.316-330. 10.1016/j.neuroimage.2017.11.051 . hal-02341960

\section{HAL Id: hal-02341960 \\ https://hal.science/hal-02341960}

Submitted on 28 Jan 2021

HAL is a multi-disciplinary open access archive for the deposit and dissemination of scientific research documents, whether they are published or not. The documents may come from teaching and research institutions in France or abroad, or from public or private research centers.
L'archive ouverte pluridisciplinaire HAL, est destinée au dépôt et à la diffusion de documents scientifiques de niveau recherche, publiés ou non, émanant des établissements d'enseignement et de recherche français ou étrangers, des laboratoires publics ou privés. 


\title{
Dynamics of perceptual decisions about symmetry in visual cortex
}

\author{
Peter J. Kohler ${ }^{a, *}$, Benoit R. Cottereau ${ }^{b, c}$, Anthony M. Norcia ${ }^{a}$ \\ ${ }^{a}$ Department of Psychology, Stanford University, Jordan Hall, Building 420, 450 Serra Mall, Stanford, CA 94305, United States \\ ${ }^{\mathrm{b}}$ Université de Toulouse, Centre de Recherche Cerveau et Cognition, Toulouse, France \\ ${ }^{\mathrm{c}}$ Centre National de la Recherche Scientifique, Toulouse Cedex, France
}

\section{A R T I C L E I N F O}

\section{Keywords:}

Symmetry

Rotation

Decision making

Electroencephalography

Temporal dynamics

\begin{abstract}
A B S T R A C T
Neuroimaging studies have identified multiple extra-striate visual areas that are sensitive to symmetry in planar images (Kohler et al., 2016; Sasaki et al., 2005). Here, we investigated which of these areas are directly involved in perceptual decisions about symmetry, by recording high-density EEG in participants $(n=25)$ who made rapid judgments about whether an exemplar image contained rotation symmetry or not. Stimulus-locked sensor-level analysis revealed symmetry-specific activity that increased with increasing order of rotation symmetry. Responselocked analysis identified activity occurring between 600 and $200 \mathrm{~ms}$ before the button-press, that was directly related to perceptual decision making. We then used fMRI-informed EEG source imaging to characterize the dynamics of symmetry-specific activity within an extended network of areas in visual cortex. The most consistent cortical source of the stimulus-locked activity was VO1, a topographically organized area in ventral visual cortex, that was highly sensitive to symmetry in a previous study (Kohler et al., 2016). Importantly, VO1 activity also contained a strong decision-related component, suggesting that this area plays a crucial role in perceptual decisions about symmetry. Other candidate areas, such as lateral occipital cortex, had weak stimulus-locked symmetry responses and no evidence of correlation with response timing.
\end{abstract}

Symmetry is a highly salient feature of the natural world, found in both plants and animals as well as human artifacts. There is a large literature investigating the role of symmetry in visual perception that has proceeded along three separate, but related lines of inquiry. First, the computational operations necessary to detect symmetry have commanded a strong interest, mainly concerned with the necessity of comparing features from spatially distant parts of the visual field (Cohen and Zaidi, 2013; Dakin and Watt, 1994). This computational demand suggests a close relationship between symmetry and global integration processes involved in the perception of contours and motion (Lorenceau and Shiffrar, 1992; McDermott and Adelson, 2004).

The second line of inquiry considers the prevalence of symmetry in natural objects, which means that symmetry can serve as a powerful cue for the detection, recognition and classification of objects. Symmetry has been shown to have strong effects on multiple aspects of object processing, such as shape discrimination (Delius and Nowak, 1982), mate selection (Møller, 1992), and shape constancy operations (Li et al., 2013). Symmetry may also influence scene perception, reducing the number of perceived elements in a scene (Apthorp and Bell, 2015).

The third line of inquiry proposes that symmetry may play a key role in neural representations, because the neural code of an input image can be made more effective by coding repeating image elements in terms of their symmetries, rather than coding each image element independently. Because symmetries are ubiquitous in the natural world, taking them into account makes for a highly efficient coding strategy (Mumford and Desolneux, 2010).

This longstanding interest in symmetry has understandably generated a strong interest in identifying the neural mechanisms supporting symmetry perception. The first push in that direction used electroencephalography (EEG) to study event-related potentials (ERPs) associated with processing of reflection symmetry, and found a differential response in posterior electrodes over occipital cortex, beginning at $\sim 220 \mathrm{~ms}$, well after the response to the contrast change evoked by the image update (Norcia et al., 2002). This initial EEG study has been followed by several others measuring similar Sustained Posterior Negativity (SPN) responses to different types of symmetric patterns (Bertamini and Makin, 2014; Jacobsen and Höfel, 2003; Makin et al., 2014, 2012, 2016; Palumbo et al., 2015). Interestingly, while SPN latency is fairly consistent across different stimuli and experiments, the amplitude has been found to scale parametrically with the strength of the symmetry in a presented image (Palumbo et al., 2015). Recent work has suggested that SPN amplitude may in fact index a more general neural response to the perceptual

\footnotetext{
* Corresponding author.

E-mail address: pjkohler@stanford.edu (P.J. Kohler).
} 
goodness of visual regularities (Makin et al., 2016).

Functional MRI studies on symmetry began with two studies published the same year, that both focused on reflection symmetry in dot patterns. The first study found a particularly strong response to symmetry in dorsal lateral occipital cortex, compared to retinotopic cortex (Tyler et al., 2005). The second study used an ROI definition procedure that was independent of symmetry responses, and found several areas in extra-striate visual cortex that were sensitive to reflection symmetry (Sasaki et al., 2005). Some areas remained sensitive even when attention was controlled - these areas included object-sensitive lateral occipital cortex (LOC), ventral V4 (adjacent to ventral V3, presumably defined as a quarter-field representation of the contralateral upper visual field, following Hadjikhani et al., 1998), V3A, V7 (commonly referred to as IPS0, see Swisher et al., 2007), and an area the authors called dorsal V4, which appears to correspond to either V3B or LO1. Another group of areas had significant responses only during passive viewing, including V3 and the motion-sensitive area MT+ (Sasaki et al., 2005).

We have recently expanded on this existing work, using a combination of EEG and functional MRI to measure responses to a novel set of stimuli comprised of wallpaper patterns (Kohler et al., 2016). Wallpapers are a class of regular textures that result from combinations of the four fundamental types of symmetry reflection, translation, rotation and glide (Fedorov, 1891; Polya, 1924). We focused on a subset of four of the 17 distinct wallpaper groups - those that contain rotation symmetries of various order. Using a combination of functional MRI and EEG source-imaging we showed that visual areas V3, hV4, VO1 and LOC had responses that varied parametrically with the order of rotation symmetry (Kohler et al., 2016). This sensitivity to rotation symmetry was not observed in V1 or V2, suggesting that the information conveyed by this form of regularity is not encoded prior to V3. EEG source-imaging allowed us to study the temporal order of symmetry processing across visual areas, and to determine that the onset of the symmetry responses occurred earlier in V3 and hV4 than in LOC. This result suggests that symmetry information is propagated along the ventral stream in a bottom-up fashion (Kohler et al., 2016).

The literature thus suggest that multiple visual areas process symmetry, but the causal role of the individual areas in symmetry perception remains poorly understood. A recent study found that reflection symmetry processing was disrupted when TMS was applied to LOC, but was unaffected when TMS was applied to V1/V2 (Bona et al., 2014; Cattaneo et al., 2011). This suggests that under the conditions of these experiments, LOC likely had a causal role in reflection symmetry perception, whereas V1/V2 did not. While these results indicate that LOC may be part of the cortical network supporting symmetry perception, they do not speak to the possible causal involvement of other areas that are responsive to symmetry, such as mid-level extra-striate areas e.g. V3, hV4 or VO1.

Here we present an EEG study in which we took a different approach to addressing the causal role of different visual areas in symmetry perception. We collected EEG measurements of the neuronal response to rotation symmetry, in the tradition of the initial ERP studies of symmetry. We expanded on the existing EEG and fMRI literature, however, by using an experiment design that made it possible to relate EEG measurements of brain activity directly to trial-to-trial variability in behavior. Our stimuli were textures from the four wallpaper groups containing rotation symmetries of varying order, that we have previously studied (Kohler et al., 2016). We asked participants to make speeded judgments about whether a presented texture image contained rotation symmetry or not. This approach allowed us to take two separate analysis approaches: Stimulus-locked analysis, which identified encoding-related activity analogous to that studied in our previous work, and response-locked analysis, which identified activity that can be directly related to the timing of button-press responses made by participants in each trial, and thus to the timing of the underlying perceptual decision making process.

We first identify a set of electrodes over occipital cortex that have the most pronounced differences in the encoding-related activity evoked by symmetry and no-symmetry stimuli, indicating that they are responding to symmetry. These electrodes also have decision-related activity that is separable from the encoding-related activity. We then use source imaging to localize both encoding- and decision-related activity in visual cortex. We can now ask which of the known symmetry-responsive visual areas show encoding-related responses to symmetry, and which of them also have activity that can be uniquely related to the perceptual decisions about symmetry.

This response-locked analysis approach has been successfully applied to investigations of the causal role of different visual areas in decisions about other perceptual domains such as shape discrimination (Ales et al., 2013) and disparity detection (Cottereau et al., 2014). Here response-locked analysis was used to determine which of the several areas that are capable of encoding symmetry are used to decode symmetry for perception. Considering that symmetry makes key contributions to multiple domains of visual perception, it is perhaps not surprising that a large number of visual areas are sensitive to symmetry. It is important, however, to clarify the contribution of each area to the ongoing perception of symmetry itself. This is the first study to approach this problem by coupling the trial-to-trial variability of brain and behavior.

\section{Materials and methods}

\section{Participants}

Twenty-five participants ( 11 females, mean age $27.8 \pm 11.7$ ) took part in the EEG experiment. Structural MRI data were collected in fifteen of these participants ( 6 females, mean age $30.8 \pm 13.6$ ), so that individualized head models could be constructed for distributed inverse source modeling. All participants were pre-screened to confirm that they had normal or corrected-to-normal visual acuity on the Bailey-Lovie chart and normal stereopsis on the RandDot test (http://precisionvision.com/products/stereo-vision-tests/randot-stereo-test.html). Their written informed consent was obtained prior to the experiment under a protocol that was approved by the Institutional Review Board of Stanford University.

\section{Wallpaper stimulus generation}

The four wallpaper groups used in this study, $P 2, P 3, P 4$ and $P 6$, all contain rotation symmetries, but the maximum order of rotation symmetry is different from group to group. Rotation symmetry around a point can be defined in terms of its order $n$, which means that the object can be rotated by an angle $360^{\circ} / \mathrm{n}$ without changing. Each group contains rotation symmetry around several points that vary in order. For P2, the maximum order of rotation symmetry is 2 , for P3 it is 3 , for P4 it is 4 and for P6 it is 6 (we encourage the reader to identify rotation symmetries of different order in Fig. 1A).

Exemplars from the different wallpaper groups were generated using a modified version of the methodology developed by Clarke et al. (2011) that we have described in detail elsewhere (Kohler et al., 2016). Briefly, exemplar patterns for each group were generated from random-noise textures, which were then repeated and transformed to cover the plane, according to the symmetry axes and geometric lattice specific to each group. The use of noise textures as the starting point for stimulus generation allowed the creation of an almost infinite number of distinct exemplars of each wallpaper group.

To control for the contribution of low level stimulus features to the measured evoked responses, phase-randomized control exemplars were generated that had the same power spectrum as each exemplar image for each group. The phase scrambling operation eliminates rotation, reflection and glide-reflection symmetry relationships, but preserves the power spectrum. As a result of the phase scrambling operation, all scrambled control exemplars, regardless of which wallpaper group they are derived from, degenerate to another symmetry group, namely $P 1$. P1 is the 


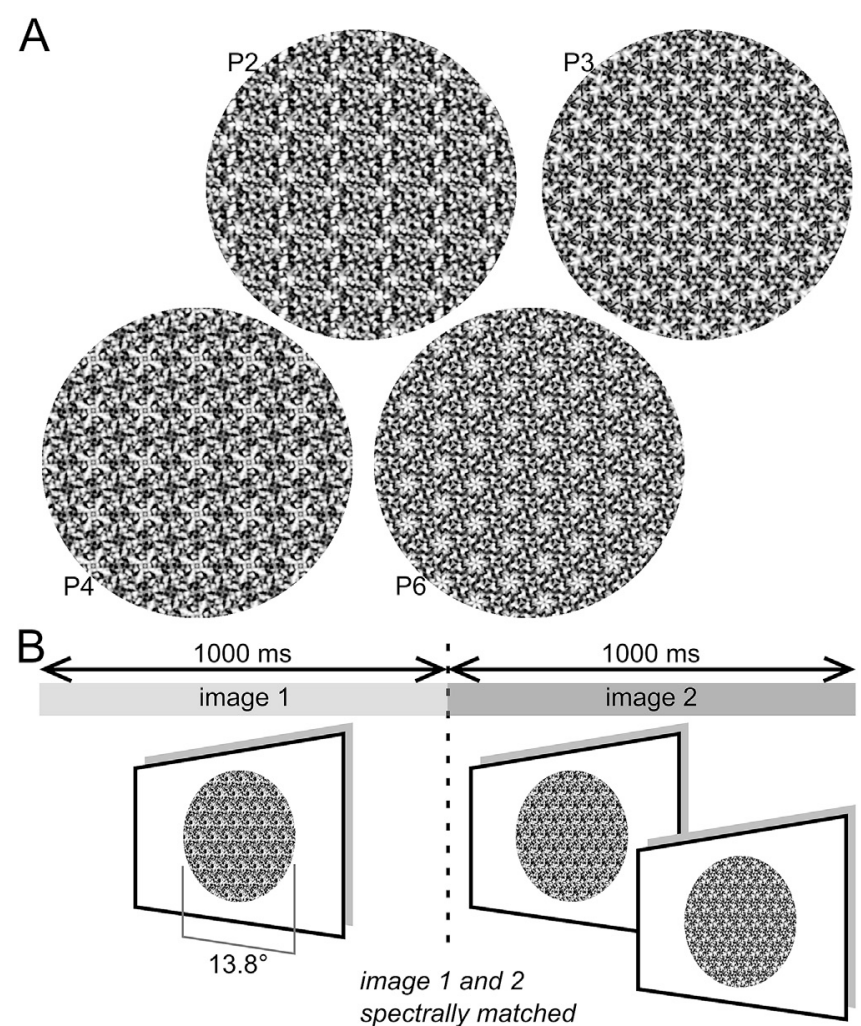

subject waits \begin{tabular}{ll|}
\hline left or right & response \\
button press & deadline: \\
\hline
\end{tabular}

Fig. 1. Experiment Stimulus and Design. (A) Exemplar images belonging to wallpaper groups P2, P3 (top row), P4 and P6 (bottom row). (B) Schematic illustrating the experimental design. Image 1 was always an exemplar image belonging to wallpaper group P1. Image 2 was either different P1 exemplar or an exemplar from one of the four groups containing rotation symmetry (PX). Images 1 and 2 were always spectrally matched. Each image was shown for $1000 \mathrm{~ms}$; participants had to indicate with a button press whether the second image was P1 or PX. If their response was more than $600 \mathrm{~ms}$ after the onset of the second image, a tone was played after the trial indicating a late response.

simplest of the wallpaper groups (Liu et al., 2010) and contains only translations of a region whose shape derives from the lattice. Because different wallpaper groups have different lattices, P1 controls matched to different groups have different power spectra. In our experiment, P2 and P4 had the same lattice, while P3 and P6 shared a different lattice. Our experimental design takes these differences into account by always comparing the neural responses evoked by each wallpaper group to responses evoked by the matched P1 control exemplars. This means that power spectrum differences between groups could not drive our effects.

\section{Stimulus presentation}

The stimuli were shown on a 24.5" Sony Trimaster EL PVM-2541 organic light emitting diode (OLED) display, with a screen resolution of $1920 \times 1080$ pixels, 8-bit color depth and a refresh rate of $60 \mathrm{~Hz}$, viewed at $70 \mathrm{~cm}$. The mean luminance was $70 \mathrm{~cd} / \mathrm{m}^{2}$ and contrast was $95 \%$. The diameter of the circular aperture was $13.8^{\circ}$ of visual angle, and the background was gray.

\section{Experimental procedure}

There were four experimental conditions, each corresponding to one of four wallpaper groups: P2, P3, P4, P6. The structure of each trial is shown in Fig. 1B: Participants were shown two successive images, with each image presentation lasting $1000 \mathrm{~ms}$, and were asked to determine if the second image contained rotation symmetry or not. The first image

was always a control image from wallpaper group P1, matched to the wallpaper group (P2, P3, P4 or P6) associated with the second image in that trial. There were two trial types per condition defined by whether the second image was the same as or different from the first image. On same trials, the second image was a different $\mathrm{P} 1$ exemplar, again matched to the current wallpaper group. This exchange led to a detectable contrast transient, but no change in group. On different trials, the second image was an exemplar belonging to one of the current wallpaper groups, P2, P3, P4 or P6, depending on the condition - for all groups, we will refer to this as PX. Prior to the experiment, participants were shown examples of the five wallpaper groups in the experiment so that they could familiarize themselves with the differences between P1 and PX. We pointed out rotation symmetry axes present in PX (and absent in P1), but participants were encouraged to look for overall structure, rather than individual symmetry axes, when doing the task.

Participants were told to respond as quickly and accurately as possible. To encourage rapid responses, participants were instructed to respond no later than $600 \mathrm{~ms}$ after the onset of the second image. If a response occurred later than $600 \mathrm{~ms}$, or not at all, this was communicated to participants with a beep sound, that was played after the end of the trial. Prior to collecting any EEG data, participants were also given several practice trials for each condition, to ensure that they understood the task and could make responses within the required time frame. Participants made responses by pressing two buttons on a game pad, using their left index finger for "same" trials and their right index finger for "different" trials.

For each condition, $10 \mathrm{P} 1$ exemplars were used as the first image, and were then paired with 10 PX exemplars (on "different" trials) and 10 different P1 exemplars (on "same" trials). For each image pair within each trial type, a differential rotation between 0 and $324^{\circ}$ was applied so that symmetry axes would not line up between trials with different exemplars. For eight of our participants, the 20 image pairs per condition were repeated 6 times, for a total of 480 trials. For the remaining 17 participants, there were 4 repetitions, for a total of 320 trials.

\section{Behavioral analysis}

We computed reaction time, percent correct and d' for all participants. Out of the twenty-five participants, two were unable to perform the task with the P2 stimulus. One participant had d' $<0$, indicating that she tended to report PX as P1, and vice versa. The other had d' $=0$ indicating that he was at chance level. Both of these participants' data, for all four conditions, were excluded from further analysis - no other participants had d' lower than 0.3 for any of the four conditions.

Although participants were instructed to respond no later than $600 \mathrm{~ms}$ after the presentation of the second image, we only rejected responses if they occurred later than $900 \mathrm{~ms}$ after the presentation of the second image - this threshold was imposed mainly to ensure that waveforms would include at least $100 \mathrm{~ms}$ of data occurring after the button press. We also rejected any trials that occurred before the onset of the second image. Both early and late responses were extremely rare, occurring on less than $1 \%$ and $3 \%$ of trials, respectively. As a result, the average number of total trials, 'Hits', 'Misses', 'Correct Rejects' and 'False alarms' was $330,140,26,127$, and 37 trials per participant, respectively. The corresponding standard deviations were equal to $65,25,17,34$ and 20. The average numbers of misses and false alarms were too small to permit a proper analysis and we therefore focus on hits and correct rejects in this study.

\section{EEG acquisition and preprocessing}

The EEG data were collected with 128-sensor HydroCell Sensor Nets (Electrical Geodesics, Eugene, OR) and band-pass filtered off-line from 0.3 to $50 \mathrm{~Hz}$. Following each experimental session, the 3D locations of all electrodes and three major fiducials (nasion, left and right pre-auricular points) were digitized using a 3Space Fastrack 3D digitizer (Polhemus, 
Colchester, VT). The digitized locations were used to construct individualized EEG forward models for 15 of the participants (see EEG SourceImaging Analysis section). Raw data were subjected to an off-line sample-by-sample thresholding procedure in which noisy sensors were replaced by the average of the six nearest spatial neighbors. On average, less than $5 \%$ of the electrodes were substituted; these electrodes were mainly located near the forehead or the ears, and as such are likely to have a negligible impact on our results, as our stimuli will likely drive responses mainly at electrodes over occipital, temporal and parietal locations. The EEG data was then re-referenced to the common average of all the sensors and segmented into $2000 \mathrm{~ms}$ epochs (lasting from the onset of image 1 until the offset of image 2). Epochs for which a large percentage of data samples exceeded a noise threshold (depending on the participant and ranging between 25 and $50 \mu \mathrm{V}$ ) were excluded from the analysis on a sensor-by-sensor basis. This was typically the case for epochs containing artifacts, such as blinks or eye movements. Across all trials and participants, $11.1 \%$ of electrodes were excluded per trial. Finally, waveforms from each trial were baseline-corrected by subtracting the temporal average of the last $100 \mathrm{~ms}$ of the first image (900-1000 ms into the trial) from the entire waveform. There was a 4.5 msec delay between the trigger for trial onset and the top of the video frame (stimulus onset). This delay has not been corrected for in the stimulus-locked analysis. We did not perform any further baselinecorrection at any stage of our analysis, and baseline-corrected data served as the input to both the response-locked analyses in sensor-space as well as the stimulus- and response-locked analyses in source space.

\section{Structural MRI acquisition}

Functional and structural MRI data were collected on a General Electric Discovery 750 (General Electric Healthcare) equipped with a 32channel head coil (Nova Medical) at the Center for Cognitive and Neurobiological Imaging at Stanford University. For each participant, we acquired two whole-brain T1-weighted structural datasets $(1.0 \times 1.0 \times 1.0 \mathrm{~mm}$ resolution, $\mathrm{TE}=2.5 \mathrm{~ms}$, TR $=6.6 \mathrm{~ms}$, flip angle $=12, \mathrm{FOV}=256 \times 256)$ and one single whole-brain T2-weighted structural dataset $(1.0 \times 1.0 \times 1.0 \mathrm{~mm}$ resolution, TE $=75 \mathrm{~ms}$, $\mathrm{TR}=2500 \mathrm{~ms}$, flip angle $=90, \mathrm{FOV}=256 \times 256$ ). These three scans were used for the tissue segmentation procedure.

\section{Tissue segmentation procedure}

The FreeSurfer software package (http://surfer.nmr.mgh.harvard. edu) was used to determine the boundaries between gray and white matter, and gray matter and cerebrospinal fluid (CSF), and generate cortical surface meshes (Dale et al., 1999). To avoid discontinuities in the cortically constrained inversion procedure arising from curvature differences between the gray/white and gray/CSF boundary, we generated a surface partway between these two boundaries that has gyri and sulci with approximately equal curvature. This "mid-gray" cortical surface consisted of a very dense triangular tessellation of several hundred thousand regularly spaced vertices. Using the MNE software package, we down-sampled this tessellation from 292,010 to 20,484 vertices using the MNE software package (http://martinos.org/mne/stable/index.html), a number high enough to accurately reflect the shape of cortical manifold (see e.g. Baillet et al., 2001), yet low enough to compute the forward model on a standard workstation. The final mid-gray surface was used to define the visual ROIs (see Atlas-based regions-of-interest section) and the source space for the EEG current modeling. Additional boundaries between the brain/CSF and the skull, the skull and the scalp, and the scalp and the air are required to generate the inverse model used for source imaging (see EEG Source-Imaging Analysis section). These boundaries were defined based on the T1-and T2-weighted structural MRI datasets, by using the FSL toolbox (http://www.fmrib.ox.ac.uk/fsl/) to segment contiguous volume regions for the inner skull, outer skull, and scalp. These MRI volumes could then be converted into inner skull, outer skull, and scalp surfaces (Smith, 2002; Smith et al., 2004) that define each of the three required boundaries.

\section{Atlas-based regions-of-interest}

Topographically organized visual ROIs were derived from a probabilistic atlas (Wang et al., 2015). The atlas was generated by functionally defining 25 topographic ROIs covering 22 visual areas in $\sim 50$ individual participants, converting the surface data from each individual to surface-based standardized space (Argall et al., 2006) and then assessing the likelihood, across participants, of any particular vector on the standardized surface belonging to a particular ROI (Wang et al., 2015). The atlas was defined using a maximum probability approach, which considers a given vector as part of the set of ROIs if it is more often found within the set, than outside the set, across participants. If this is the case, the vector is then assigned the value of the most likely ROI, and if not, it was considered to be outside the set of ROIs. The maximum probability approach captures much of the overall structure of ROIs defined for individual participants and generalizes well to novel participants who did not contribute to the atlas generation (Wang et al., 2015). We downloaded the atlas from http://scholar.princeton.edu/sites/default/files/ napl/files/probatlas_v4.zip and converted the ROIs from standardized surface space to individual surface space for each of our participants, using nearest-neighbor interpolation. We then used surface-based clustering to eliminate vertices more than 1 edge removed from the main cluster of each ROI, to ensure that all ROIs consisted exclusively of contiguous vertices. This step eliminated small speckles, while having minimal effect on the overall structure and extent of the ROIs. Finally, the ROIs were down-sampled to match the 20,484 vertex surface mesh used for source localization.

\section{EEG sensor space analysis}

For the EEG sensor-space analysis, we defined sensor ROIs, consisting of the 16 electrodes (out of 128) that had the longest run of consecutive time points with $p$-values less than 0.05 for a one-tailed paired $t$-test comparing PX and P1, where PX and P1 were within-participant averages across the four wallpaper groups. We split the 23-participant data set into odd and even halves, and defined sensor ROIs separately for each half. We then used each of the two 16-electrode ROIs to inspect the other half of the data, so that ROI definitions were independent of the data being analyzed. By selecting the features to include in the analysis (in this case: electrodes) based on data that are independent from the data being analyzed, this procedure avoids so-called "double dipping" - the use of the same data set for selection and selective analysis - which can result in distorted descriptive statistics and invalid statistical inference (Kriegeskorte et al., 2009).

For the stimulus-locked analysis of response-timing and the responselocked comparison of intact and shuffled waveforms, our goal is not to identify differences between PX and P1 waveforms, the criteria we used for feature selection, so it is not necessary to analyze odd and even datasets separately. Thus, we analyzed data from all 23 participants as a single dataset, using the 12 electrodes that were shared between the two sensor ROIs defined based on each half of the stimulus-locked data. We generated response-locked waveforms by selecting time points over a window from $1000 \mathrm{~ms}$ prior to the button press until $100 \mathrm{~ms}$ after the button press. When these waveforms are averaged together, the times of the button presses will be aligned, although button presses occurred at different times during each trial.

\section{EEG Source-Imaging Analysis}

For each participant, the EEG source space was defined by the midgray surface (see Tissue segmentation procedure section). The distance between the vertices of this surface was on average $3.7 \mathrm{~mm}$, with a standard deviation of $1.5 \mathrm{~mm}$, range $0.1-11 \mathrm{~mm}$. Current dipoles were 
placed at each of these vertices. Their orientations were constrained to be orthogonal to the cortical surface to diminish the number of parameters to be estimated in the inverse procedure (Hämäläinen et al., 1993). The source space, the 3D electrode locations, and the individually defined boundaries were then combined using the MNE software package to characterize the electric field propagation using a three-compartment boundary element method (Hämäläinen and Sarvas, 1989). The resulting forward model is linear and links the activity of the 20,484 cortical sources to the voltages recorded by our EEG electrodes.

Cortical current density estimates of the neural responses were obtained from an L2 minimum-norm inverse of the forward model as described by Cottereau et al. (2012). We used the atlas-defined visual ROIs (see Atlas-based regions-of-interest section) to constrain these estimates by modifying the source-covariance matrix (functional area constrained estimation or FACE). The aim of this procedure was to decrease the tendency of the minimum-norm procedure to smooth activity across different visual ROIs. Two modifications were applied: 1) we increased the variance allowed within the ROIs by a factor of two relative to other vertices; and 2) we enforced a local correlation constraint within each ROI using the first- and second-order neighborhoods on the cortical tessellation with a weighting function equal to 0.5 for the first order and 0.25 for the second. This correlation constraint, therefore, respects both retinotopy and boundaries between visual areas, and permits a more precise dissociation of signals from different ROIs. This is not the case for other smoothing methods, such as LORETA, that apply the same smoothing rule across all of cortex (Pascual-Marqui et al., 1994). For each participant, condition and trial type, the FACE inversion scheme was applied to waveforms generated by averaging across each epoch (see $E E G$ Acquisition and Preprocessing section), which resulted in an estimation of these waveforms for each source on the cortical tessellation. The waveforms were then averaged across all the sources belonging to each visual ROI, for each participant, to determine the average response for each ROI, which were in turn averaged across participants. The FACE procedure has been shown to reduce cross-talk between different cortical ROIs resulting in better area resolution in the group inversion than can be achieved in single participants (Cottereau et al., 2015).

\section{Comparison of amplitude differences over time}

Differences in response amplitude between the experimental conditions were identified by a permutation test based on methods devised by Blair and Karniski (1993) and described in detail by Appelbaum et al. (2006). Briefly, this approach tests the null hypothesis that no differences were present between experimental conditions tested by making synthetic data sets in which the two condition labels were randomly permuted across participants. For the stimulus-locked sensor space analysis ( $\mathrm{n}=11$ and $\mathrm{n}=12$ for the two data splits), synthetic data sets were generated for all possible permutations (2048 and 4096, respectively). For the stimulus-locked analysis of response timing $(n=23)$ and the response-locked comparison of intact and shuffled waveforms, both performed in sensor space, 5000 synthetic data sets were generated, with permutations randomly selected among the $8,388,608$ possible permutations. The same approach was taken for the source-localization data $(n=15)$ : 5000 permutations were randomly selected among the 32,768 that were possible. For every permutation, we computed $t$-scores of the waveform difference, and found the longest run of consecutive time points with $p$-values less than 0.05 . This procedure generates a non-parametric reference distribution of consecutive significant $p$-values. We then rejected the null hypothesis if the length of any consecutive sequence of significant $t$-scores in the original, non-permuted data exceeded $95 \%$ of the values in the null distribution. Because each permutation sample contributes only its longest significant sequence to the reference distribution, this procedure implicitly compensates for the problem of multiple comparisons, and is a valid test for the omnibus hypothesis of no difference between the waveforms at any time point. Furthermore, this test not only detects significant departures from the null hypothesis, but also localizes the time periods when such departures occur. However, since the correction procedure is tied to the length of the data and the somewhat arbitrary choice of keeping family-wise error at $5 \%$, we therefore also present the uncorrected significance values (see red/yellow color maps in Figs. 3, 6, 7, 8, 10). By applying both statistical approaches, we are better able to identify time periods when the responses depart from the null hypothesis.

\section{Onset detection procedures}

We identified onsets of the stimulus-locked activity specific to symmetry using the procedure described by Osman et al. (1992), in which the onset is identified as the time-point at which the waveform achieves an amplitude consistently above or below a criterion. We used the same criterion throughout: 2.5 times the standard deviation over time, of a noise distribution that was computed over a baseline window covering the last $500 \mathrm{~ms}$ of the presentation of the first image. To be considered consistently above or below criterion, the next two 50-ms windows following the onset time-point had to exceed the criterion. Image update responses manifested as positive transients, so we estimated the onset of the image update response as the first time-point, following the presentation of the second image, for which the within-participant average of PX and P1 was consistently above criterion. Symmetry responses, on the other hand, were seen as sustained activation that was more negative for PX than P1, and were thus estimated as the first time-point for which the difference waveform generated by subtracting P1 from PX was consistently below criterion. We did not compute a common noise distribution across all conditions - for every set of waveforms that went into a given onset estimate, we computed independent noise distributions over the baseline window.

We used a jackknife procedure to estimate the standard error of the onset estimates and to quantify the significance of differences between estimates, by computing average waveforms across all participants except one, and then applying the onset detection procedure to the resulting waveform. For visualization purposes, we estimated the standard error of average onsets across all participants, based on our jackknifed onsets (Equation (2); Miller et al., 2009). We computed $t$-statistics for a two-tailed test comparing a pair of onsets, by subtracting the jackknifed onset estimates for onset $\mathrm{A}$ and $\mathrm{B}$, and then estimating the standard error of the difference (see Equation (2); Miller et al., 1998). We then subtracted onset A and B, estimated from the overall sample and divided the overall difference with the estimated standard error of the difference to derive a $t$-statistic (Equation (3); Miller et al., 1998).

\section{Results}

\section{Behavioral results}

We first examined participants' behavioral responses during the rotation symmetry detection task. As described in the Methods, we rejected two participants who were unable to do the task for group P2. For the remaining 23 participants, we then computed the average reaction time and ratio of correct responses, considering both PX and P1 trials, as well as d', for each of the four conditions. The average reaction time was $520 \mathrm{~ms}$ (standard deviation: $70 \mathrm{~ms}$ ), the average number of correct responses was $80.8 \%$ (standard deviation: $8.2 \%$ ) and the average d' was 1.97 (standard deviation: 0.69). The average reaction time, percent correct and d' are shown as bar graphs in Fig. 2.

Performance was quite comparable across the four conditions, with slightly worse accuracy and slower reaction time for P2 compared to the other 3 groups. We tested these effects with a repeated measures analysis of variance (rANOVA), implemented in R (R Core Team, 2014), using the "ez" package (Lawrence, 2015). We performed separate rANOVAs for each of the three measures of performance, and found significant main effects of condition on reaction time $(F(3,66)=12.2$, p $<0.0001)$, accuracy $(F(3,66)=8.65, \mathrm{p}<0.0001)$ and $\mathrm{d}^{\prime}(F(3,66)=11.02$, 

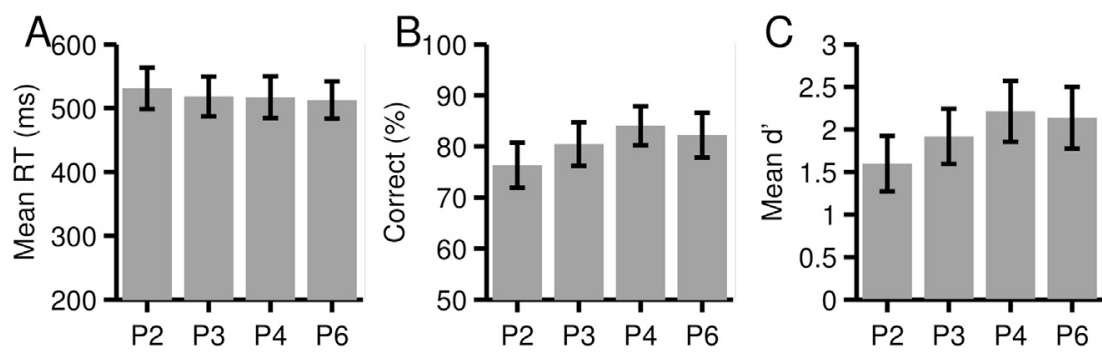

Fig. 2. Behavioral Performance. Average reaction time (A), correct responses (B) and d' (C) across 23 participants. Error bars are standard error of the mean.

$\mathrm{p}<0.0001$ ). For all three tests, Mauchly's test indicated that sphericity had not been violated. It is important to emphasize that effect sizes, computed as generalized eta squared $\left(\eta_{G}^{2}\right)$, following Bakeman's recommendation (Bakeman, 2005), were quite small: RT: 0.0097; Accuracy: 0.087; d': 0.092).

\section{Sensor level activity locked to the stimulus onset}

As mentioned above, our analysis focused exclusively on correct trials. We identified sensor ROIs separately for odd and even participants as described in the EEG sensor space analysis section. The selected electrodes were located over the back of the head, and there was a reasonable amount of overlap between the two sensor ROIs; 12 out of 16 electrodes were found in both sets (see Fig. $3 \mathrm{~A}$ and B). The responses were also quite similar across the odd and even participants, with a transient above-zero image update response occurring for both PX and P1, followed by a sustained negativity (see Fig. 3C and D).

We identified the onset of the image update response as the first timepoint for which the within-participant average of the PX and P1 waveforms, was consistently above criterion (see Onset Detection Procedures section) - this occurred at $\sim 100 \mathrm{~ms}$ after the onset of image 2 . We identified the onset of the symmetry response as the first time point for which the PX-P1 difference waveform was consistently below criterion this occurred at $\sim 150 \mathrm{~ms}$ after the onset of image 2 . There were significant differences between PX and P1 during the sustained negativity, which began at the onset of the symmetry response, and persisted until the end of the trial, consistent with prior observations (Makin et al., 2016; Norcia et al., 2002). Not surprisingly, given its long duration, this run of differences survived the permutation-based correction procedure. The clear majority of button-presses, indicated in gray on Fig. 3C and D, occurred during this period. Both the odd and even halves of the data exhibited this difference, and the onset estimates were also very similar.

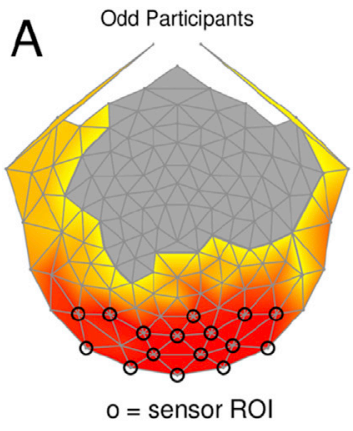

B Even Participants

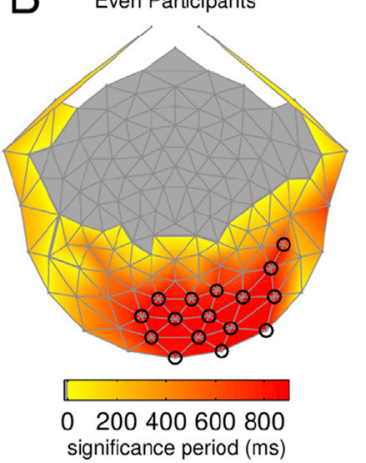

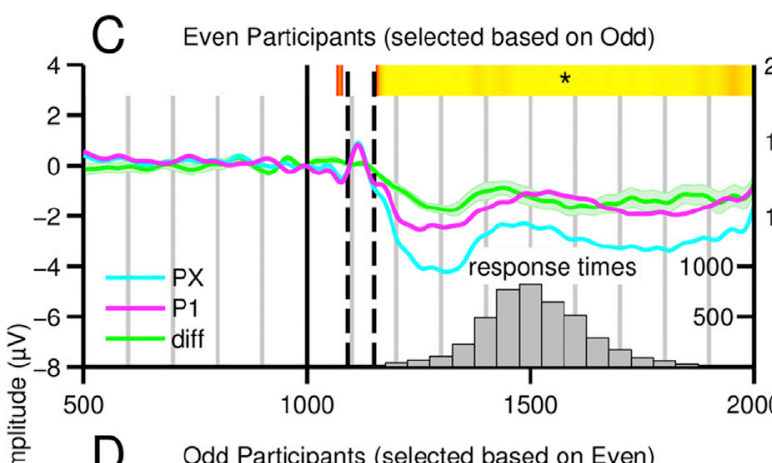

onset delay (ms)
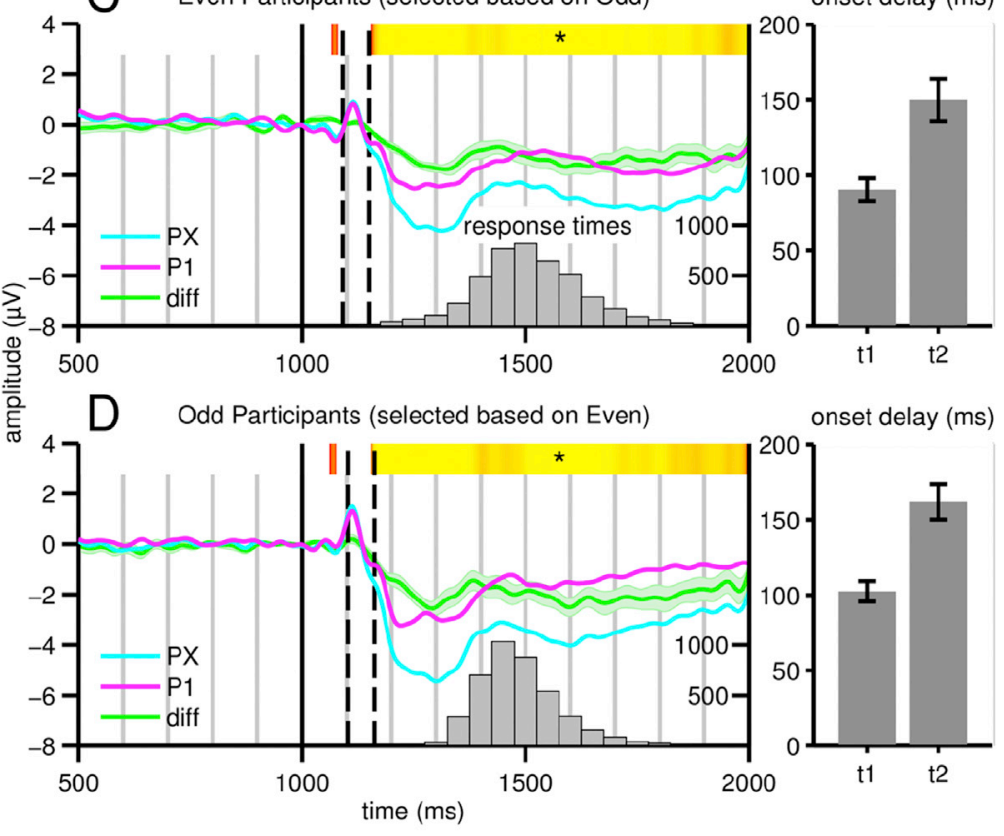

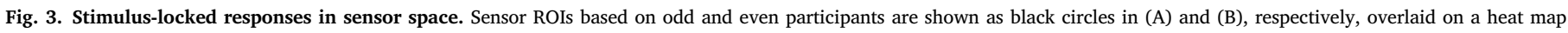

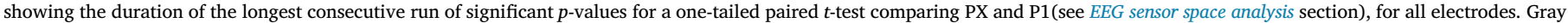

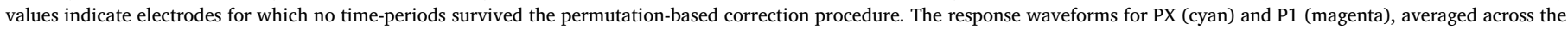

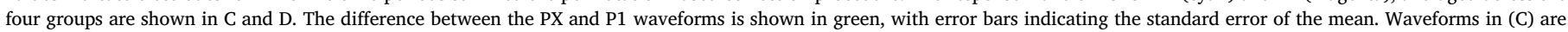

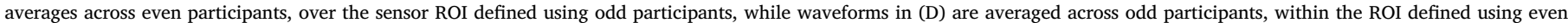

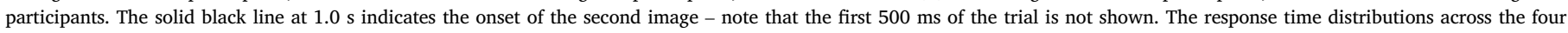

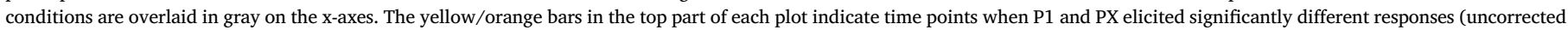

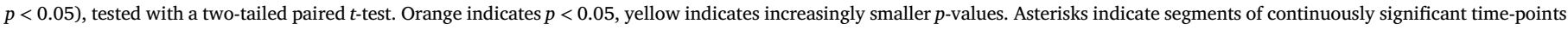

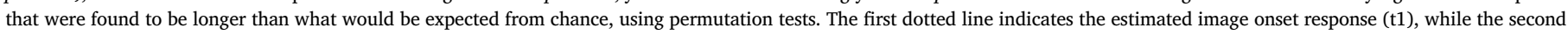

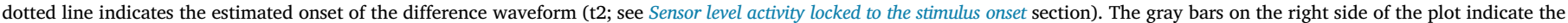
onset delays in ms after the presentation of the second image, with error bars estimated using a jack-knife procedure (see section Comparison of amplitude differences over time). 
We also repeated the symmetry onset estimation and PX and P1 comparison for P2, P3, P4 and P6 separately. These results mostly replicated our previous finding that visual responses to wallpaper textures increase parametrically with the maximum order of rotation symmetry within the groups (Kohler et al., 2016). P2 (maximum order $=2$ ) and P3 (maximum order $=3$ ) yielded smaller, and less consistently significant differences between PX and P1, than P4 (maximum order = 4) and P6 (maximum order $=6$ ). Symmetry response onsets were fairly stable across groups, with the exception of P2 for the odd half of the P2 data, where the onset could not be detected until very late, as a result of PX and P1 not being reliably different (see Supplementary Fig. S1).

To visualize the pattern of activity evoked across all electrodes, as symmetry processing unfolds, we plotted electrode maps of responses to $\mathrm{PX}$ and $\mathrm{P} 1$ at 3 different time-points: The image onset (t1), the symmetry onset ( $\mathrm{t} 2$ ) and the peak symmetry response ( $\mathrm{t} 3$, identified as the maximum difference between P1 and PX). Since these maps are for visualization only, we collapse over the two split halves of the data (see Fig. 4). We see little difference between PX and P1 at the image onset, a medially localized difference at the symmetry onset, which becomes more widespread laterally and ventrally at the peak symmetry response.

Supplementary video related to this article can be found at https:// doi.org/10.1016/j.neuroimage.2017.11.051.

In conclusion, our stimulus-locked sensor space analysis shows that symmetry responses begin approximately $50 \mathrm{~ms}$ after the onset of the contrast change-driven image onset response. Differential activity related to symmetry then persists for the duration of the symmetry image presentation. There is evidence of a symmetry response for all four groups tested, but P4 and P6 yield the most consistent responses. All of these effects replicate across the two independent groups of participants.

\section{Source level activity locked to the stimulus onset}

For the characterization of the data in source space, we first averaged responses to PX and P1 across the four wallpaper groups to increase the signal-to-noise ratio. We focused our analysis on 9 regions-of-interest (ROIs), extracted from a probabilistic atlas (Wang et al., 2015). We had previously identified five regions as having significant parametric responses to rotation symmetry (dorsal and ventral V3, hV4, VO1, and LOC). All five were included in current analyses, but instead of using a functionally-defined LOC ROI, we used more specific, retinotopically-defined areas LO1 and LO2. LO1 and LO2 are part of the object-selective lateral occipital complex and have significant overlap with functionally defined LOC (Larsson and Heeger, 2006). In a re-analysis of the data from our previous fMRI experiment (Kohler et al., 2016), we found that the parametric response to rotation symmetry we observed in LOC was in fact driven mostly by responses in LO1 (data not shown). Based on these data, we reasoned that using LO1 and LO2 instead of LOC could potentially yield more specific results. We also included VO2, a neighboring area to VO1 (Brewer et al., 2005) that was not analyzed in our previous study. Like the other areas that were found to be sensitive to symmetry, VO2 is positioned along the ventral object-processing pathway, and thus likely to have similar sensitivity. Finally, we included two control areas (V3A and V3B); retinotopic regions of the dorsal pathway that are not expected to be selective to symmetry, and did not have parametric responses in our previous study (Kohler et al., 2016). All of the areas used are shown in Fig. 5.

The time-courses estimated in these ROIs from our source-imaging approach are shown in Fig. 6. For both P1 and PX conditions, each of the ROIs had strong sustained responses that arose $\sim 150 \mathrm{~ms}$ after the second stimulus onset and lasted for at least $500 \mathrm{~ms}$ (see the magenta and cyan time-courses respectively corresponding to the P1 and PX conditions). In most of the ROIs, this sustained response was preceded by a transient image update response, which we also observed in the sensor space data. The differential symmetry-specific response (see the green time-courses) was significant in both sub-areas of V3 (dorsal and ventral) and in VO1 (see Fig. 6) starting $\sim 150 \mathrm{~ms}$ after the onset of the second image. This result is in line with our previous study (Kohler et al., 2016). We also found significant differential responses in area VO2. This result is new because we did not investigate VO2 in our previous study. Symmetry-specific activity in the VO2 ROI reinforces the idea that symmetry relationships are processed along the human ventral pathway. In hV4, we observed differences between responses to the P1 and PX conditions. However, these differences did not reach significance using our criteria. This result is rather surprising given that we had found strong symmetry sensitivity in this ROI in our previous study (Kohler et al., 2016). Nonetheless, as we shall see in the following sections, our response-locked analysis did identify hV4 responses that were significantly modulated by perceptual decisions about symmetry. We did not observe the sensitivity to rotation symmetry that we have previously observed in functionally defined LOC (Kohler et al., 2016), in either of its retinotopic counterparts $\mathrm{LO} 1$ and LO2. This discrepancy could potentially arise from differences in task between the previous experiment and the current one. Finally, as predicted, we saw no symmetry selectivity in our two control ROIs (V3A and V3B).

In conclusion, our stimulus-locked source-space analysis identifies significant symmetry-specific responses in both ventral and dorsal V3, as well as in ventral areas VO1 and VO2. A trend can be observed in area hV4, but did not reach significance. We see no evidence of symmetry responses in either of the two lateral occipital areas (LO1 and LO2), nor in our two control ROIs (V3A and V3B).

\section{Relationship between response time and sensor-level stimulus-locked activity}

Perceptual discriminations involve a chain of processes starting from encoding of the stimulus attributes and ending with the generation of an appropriate motor response (DiCarlo and Maunsell, 2005). The stimulus-locked analysis demonstrated that the patterns used in our experiment evoke responses that are tightly coupled to the time of target presentation and that differentiate PX from P1. These differential evoked responses begin well before the behavioral responses that are indicative of successful discrimination.

There is considerable variability in the response time (RT) for target identification (see histogram below waveforms in Fig. 3). This variability could come from a number of sources, including the initial encoding stage and/or the later processes that accumulate sensory evidence for a decision (Gold and Shadlen, 2007). The stimulus-locked analysis will emphasize RT-dependent variability that is due to the encoding process. We test for the presence of RT-dependent variability, by splitting the stimulus-locked waveforms by RT, and analyzing each group separately. If the waveforms contain encoding-related variability that contribute to RT, it should manifest as a difference between the groups.

For the stimulus-locked analysis of response-timing, data from all 23 participants were treated as a single dataset, and averaged over the 12 electrodes that were shared between the two sensor ROIs that we defined based on stimulus-locked data from the odd and even participants, respectively. For each participant, we split the stimulus-locked PX and P1 trial data into three groups, based on the button press timing: fast, medium and slow responses (Cottereau et al., 2014). The data was split using individualized criteria for each participant to ensure that each group had the same number of trials. Fig. 7 replots the stimulus-locked data from Fig. 3, collapsing across all 23 observers within the 12-electrode sensor-ROI. The data are split by RT for both PX and P1. We tested for RT-dependent differences using a paired $t$-test comparing fast and slow waveforms, and corrected for multiple comparisons with the same permutation approach that was used for the comparison between PX and P1 in the previous analysis (described in detail in the Comparison of amplitude differences over time section). There is some evidence of RT-dependent differences in the stimulus-locked data, most prominently at around $\sim 200 \mathrm{~ms}$ after the onset of the second image. This effect occurs in PX trials approximately $50 \mathrm{~ms}$ after the initial encoding of the stimulus category (see Fig. 3) and appears to be driven by a difference in slope between fast and slow trials. Because the effect does not survive 

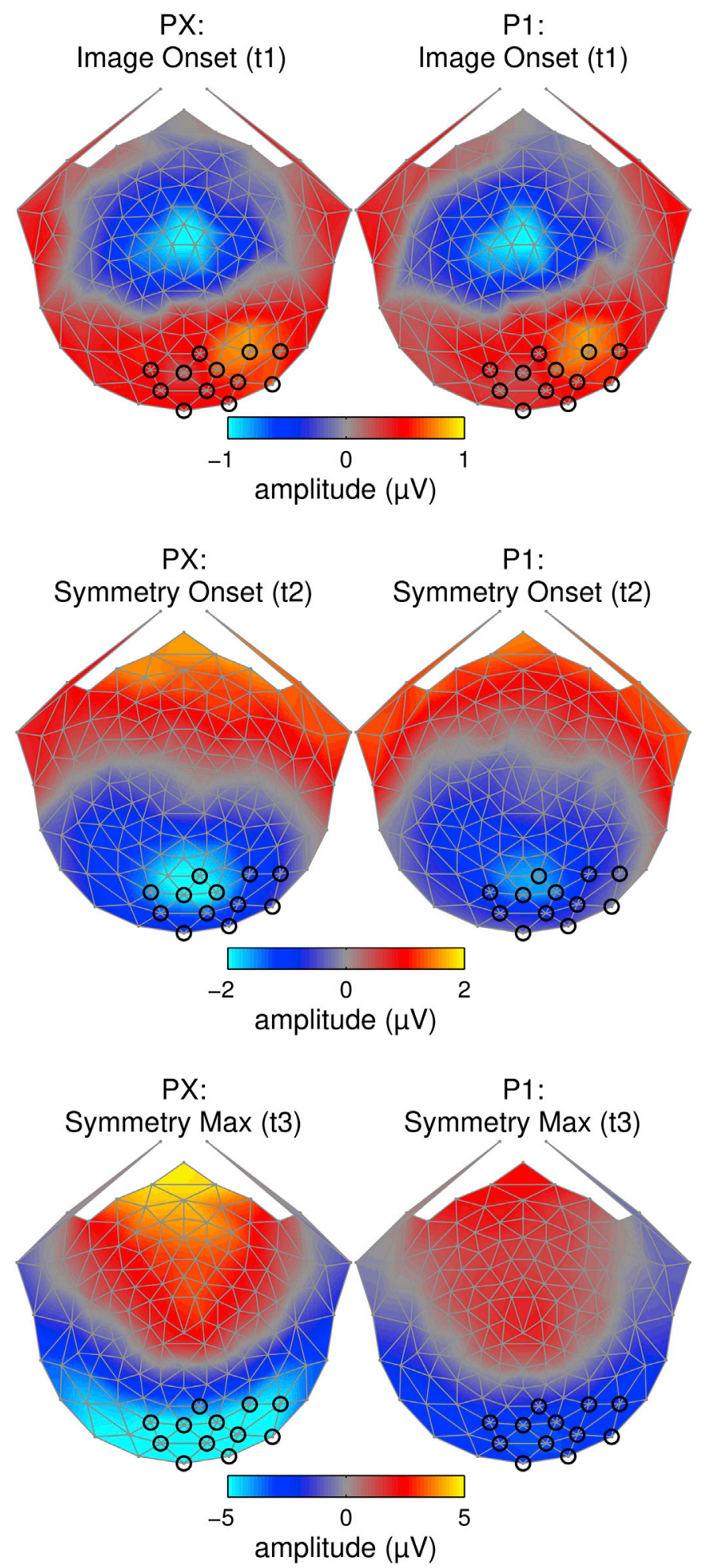

Fig. 4. Stimulus-locked activity across all electrodes as symmetry processing unfolds. Topographic maps of stimulus-locked potentials evoked by PX and P1, averaged across the four wallpaper groups, across all electrodes at three time points: (a) t1, the image onset response, occurring at $96 \mathrm{~ms}$ after the presentation of the second image; (b) $\mathrm{t} 2$, the symmetry onset response, occurring at $156 \mathrm{~ms}$; (c) t3, the peak symmetry response, occurring at $307 \mathrm{~ms}$. The intersection of the sensor ROIs generated for the odd and even participants is overlaid on the maps - note that these maps are averages across all 23 participants. A more complete picture of the development of the stimulus-locked symmetry-specific activity over time can be observed in Supplementary Video 1, which shows equivalent topographic maps for PX and P1 over the entire duration of the second image, in $5 \mathrm{~ms}$ increments. correction for multiple comparisons it can only be considered weak evidence of encoding-related, RT-dependent variability. In the next section, we will use response-locked analysis to identify more robust decision-related, RT-dependent variability in our data.

\section{Response-locked sensor-level activity}

We can emphasize activity that is related to the accumulation of sensory evidence for a decision (Gold and Shadlen, 2007), by averaging backwards in time from the moment of the button press. This response-locked analysis will preferentially emphasize activity that has a consistent timing relationship to the behavioral decision and motor response over activity that has a consistent time relationship to the stimulus onset (DiCarlo and Maunsell, 2005). Because stimulus-locked responses to symmetry are very sustained (see Fig. 7), the response-locked analysis will contain residual low-frequency stimulus-locked components that survive the blurring generated by realigning the waveforms to the moment of the button-press. To determine whether there is variability in the response-locked waveforms that goes beyond any residual stimulus-locked activity, we repeated the response-locked analysis using RT labels that were randomly shuffled among all of the trials. This eliminates the correlation between individual trial waveforms and RT. Any differences we observe between intact and shuffled response-locked waveforms are thus directly related to decision-making.

For the response-locked analysis, we averaged response-locked intact and shuffled waveforms from all 23 participants over our combined 12electrode sensor-ROI. We then tested for differences between the intact and shuffled response-locked waveforms using a paired $t$-test, and corrected for multiple comparisons with the same permutation approach used in previous analyses (described in detail in the Comparison of amplitude differences over time section). Although the intact and shuffled waveforms are broadly similar, due to the sustained stimulus-locked component present in both, we see significant differences for PX that survive correction for multiple comparisons. The first differences occur as early as $\sim 550 \mathrm{~ms}$ prior to the button press.

Interestingly, we only see evidence of decision-related activity for P1 at the time of the button-press, where some time points also reach significance for PX (but do not survive correction). This may reflect the imbalance of the task: The first image was a P1 image in all trials, so in P1 trials a representation of the symmetries in the second (P1) image may already exist. In PX trials, the second image contain new symmetries, so a new representation must be built. In any case, the results from the PX trials demonstrate that the response-locked waveforms contain decisionrelated activity beyond that driven by the stimulus, that precedes the RT by more than $500 \mathrm{~ms}$. This activity can only be captured by taking the timing of the button-press into account and by discounting sustained stimulus-locked activity via the comparison with the shuffled waveform.

\section{Response-locked activity across all sensors}

We observed decision-related activity in our combined sensor-ROI, which was defined by selecting the electrodes that had the most significant stimulus locked, encoding-related activity. This sensor-ROI was over occipital visual areas, and yet had decision-related activity, as evidenced by the response-locked analysis. However, we also wanted to investigate the timing of decision-related activity more broadly and observe the transition to activity associated with the motor response. To do this, we plot the response-locked scalp topographies at 3 key timepoints: $400 \mathrm{~ms}$ and $200 \mathrm{~ms}$ prior to the button-press, and at the moment of the button-press. Because the intact response-locked topographies will be dominated by the residual stimulus-locked activity that is likely encoding-related, we also plot the difference between intact and shuffled waveforms, which highlights the decision-related activity (see Fig. 9).

Prior to the button-press, the topographies with intact RT assignments 


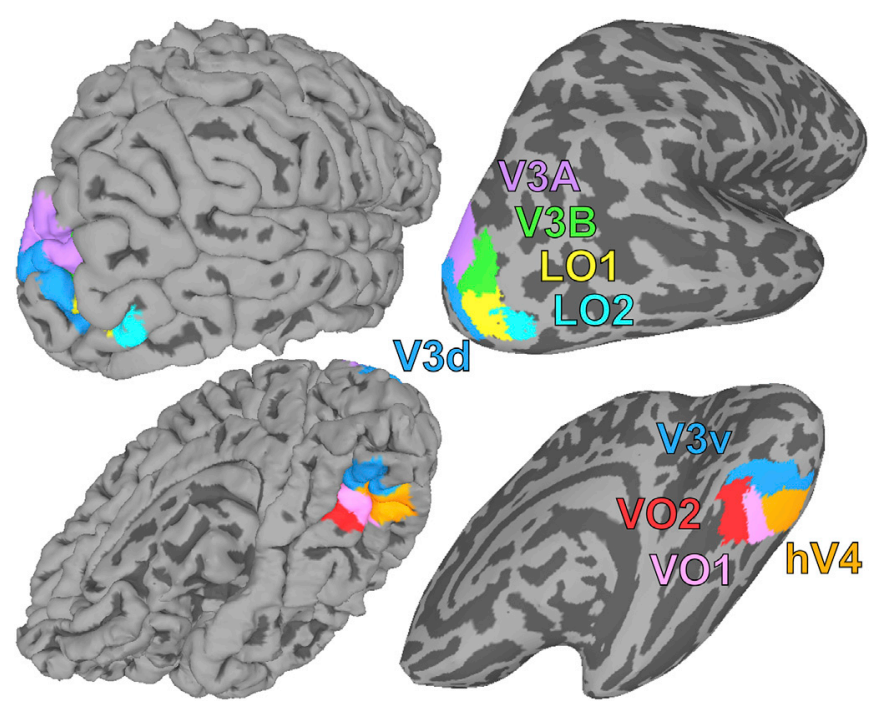

Fig. 5. Atlas-defined ROIs used for source imaging. On the left side of the figure, the ROIs are plotted on the pial surface of the right hemisphere of a single subject. On the right side of the figure, the same ROIs are shown on an inflated surface, which makes ROIs hidden inside sulci visible.

are dominated by symmetry-specific activity in visual cortex, which shows little or no difference between PX and P1 at $400 \mathrm{~ms}$ (see top row of Fig. 9A), but a large difference at $200 \mathrm{~ms}$, near the peak of the responselocked waveform (see middle row of Fig. 9A). The topographies of the difference between intact and shuffled data exhibit a broad negative difference at $400 \mathrm{~ms}$ for PX, but not P1 (see middle row of Fig. 9B). This difference includes electrodes over occipital cortex, consistent with the sensor ROI results (see Fig. 8), but is centered more anteriorly, possibly due to the involvement of sources outside visual cortex. By the time of the button-press, the intact and difference topographies are more similar (see bottom row of Fig. 9), likely reflecting a mixture of sustained encodingrelated activity and response-related activity near the time of the buttonpress. By comparing the intact vs shuffled trials (see bottom row of Fig. 9B), we eliminate the sustained stimulus-locked component associated with encoding, and now the responses for both PX and P1 are centered over central electrodes and are mirror symmetric consistent with the finger used for the button-press (right index finger for PX, left index finger for P1). The scalp topography thus clearly distinguishes two phases of the response-locked waveform, an initial phase that is dominated by stimulus-locked activity in visual areas, followed by a late phase reflecting the shift to motor response generation based on the participant's choice. As demonstrated by the sensor ROI analysis (see Fig. 8), we can identify decision-related activity during both phases.

\section{Response-locked source-level activity}

We have now identified stimulus-locked responses to symmetry both at the sensor-level and at the source-level in a subset of visual ROIs. We have also demonstrated that our response-locked analysis can identify decision-related activity that is not captured by the stimulus-locked analysis, and that is directly tied to trial-to-trial variability in participants' responses. As a final analysis, we performed our response-locked analysis on the source-level data in order to localize our decisionrelated activity to one or more visual ROIs. We conducted this analysis on the ROIs for which we saw a stimulus-locked symmetry response: V3d, $\mathrm{V} 3 \mathrm{v}, \mathrm{VO} 1$ and VO2. Although we did not find significant differences between activity evoked by the PX and P1 conditions in hV4, LO1 and LO2 (see above), we also included these ROI in the analysis, because the strong symmetry responses we previously observed in those areas (Kohler et al., 2016) made it interesting to determine whether their activity could be linked to perceptual decision.

The response-locked analysis identified decision-related activity in most of the ROIs that had exhibited significant symmetry responses in our stimulus-locked analysis (see Fig. 10). In both V3v, V4, VO1 and VO2 we found significant differences between the intact and shuffled response-locked waveforms for PX, with VO1 exhibiting the earliest consistent differences. In V3d, the differences were extremely weak and did not survive correction for multiple comparisons, which suggests that the response-locked activity seen in V3v may be a result of mislocalized sources from nearby ventral areas. As in the sensor-level analysis, there were no consistent differences between intact and shuffled P1 data prior to the button-press in any area, but LO2 exhibited significant differences at or immediate after the button press for both PX and P1. This shows that under the conditions of this experiment, LO1 and LO2 have neither encoding-related or decision-related activity, but LO2 may play a role post-decision, potentially by providing a confidence signal.

\section{Discussion}

This set of EEG results comprise the first brain imaging investigation of the neuronal activity driving perceptual decisions about symmetry. As in our previous study (Kohler et al., 2016), the stimuli were exemplars from four wallpaper groups containing rotation symmetry. The addition here of a speeded task involving the detection of the presence or absence of rotation symmetry builds on both our previous data and previous EEG investigations of symmetry by allowing us to distinguish encoding- and decision-related activity. We find that while multiple visual areas have symmetry-specific encoding-related activity, only some of them are read-out for decision. Taken together, these findings provide a detailed picture of the dynamics and likely origin in occipital cortex of neural activity driving perceptual decisions about symmetry.

\section{Decision-related activity in occipital cortex}

Our response-locked analysis of the sensor space data from occipital electrodes revealed activity that can be directly related to participants' decisions about the presence or absence of symmetry beginning around $550 \mathrm{~ms}$ before the button press (see Fig. 8). The response-locked waveforms contain large amounts of residual stimulus-locked activity, due to the highly sustained nature of the symmetry responses, but our comparison of intact and shuffled response-locked data identifies RTdependent variability in PX trials, that can only be captured by taking the timing of the button-press into account. This contrasts with the results of our stimulus-locked analysis conditioned on response timing where the RT-dependent waveform differences were weaker and did not survive corrections for multiple comparisons (see Fig. 7). This shows that the trial-to-trial variability in RT is more closely associated with the accumulation of evidence leading to a decision, rather than the initial encoding of the visual stimulus.

We can distinguish two phases of the response-locked waveforms that have different response topographies: An initial phase that is dominated by encoding-related activity in visual areas, followed by a phase dominated by motor-related responses (see Fig. 9). Importantly, we observe decision-related activity prior to the button-press that occurs during the initial phase, consistent with variability in the accumulation of sensory evidence leading to the decision, prior to the generation of the motor response. Importantly, this decision-related activity was found in a set of electrodes over occipital cortex that were selected purely based on their stimulus-locked response. Decision-related responses to symmetry thus occur over brain areas that also have strong co-localized stimulus-locked, encoding-related activity, as we have previously shown for decisions with other stimulus regimes (Ales et al., 2013; Cottereau et al., 2014).

The source-level analysis provides further evidence for decisionrelated activity in occipital visual areas. Of the 9 visual areas examined in the stimulus-locked analysis, VO1 had the most robust encodingrelated responses to rotation symmetry (see Fig. 6). Importantly, VO1 

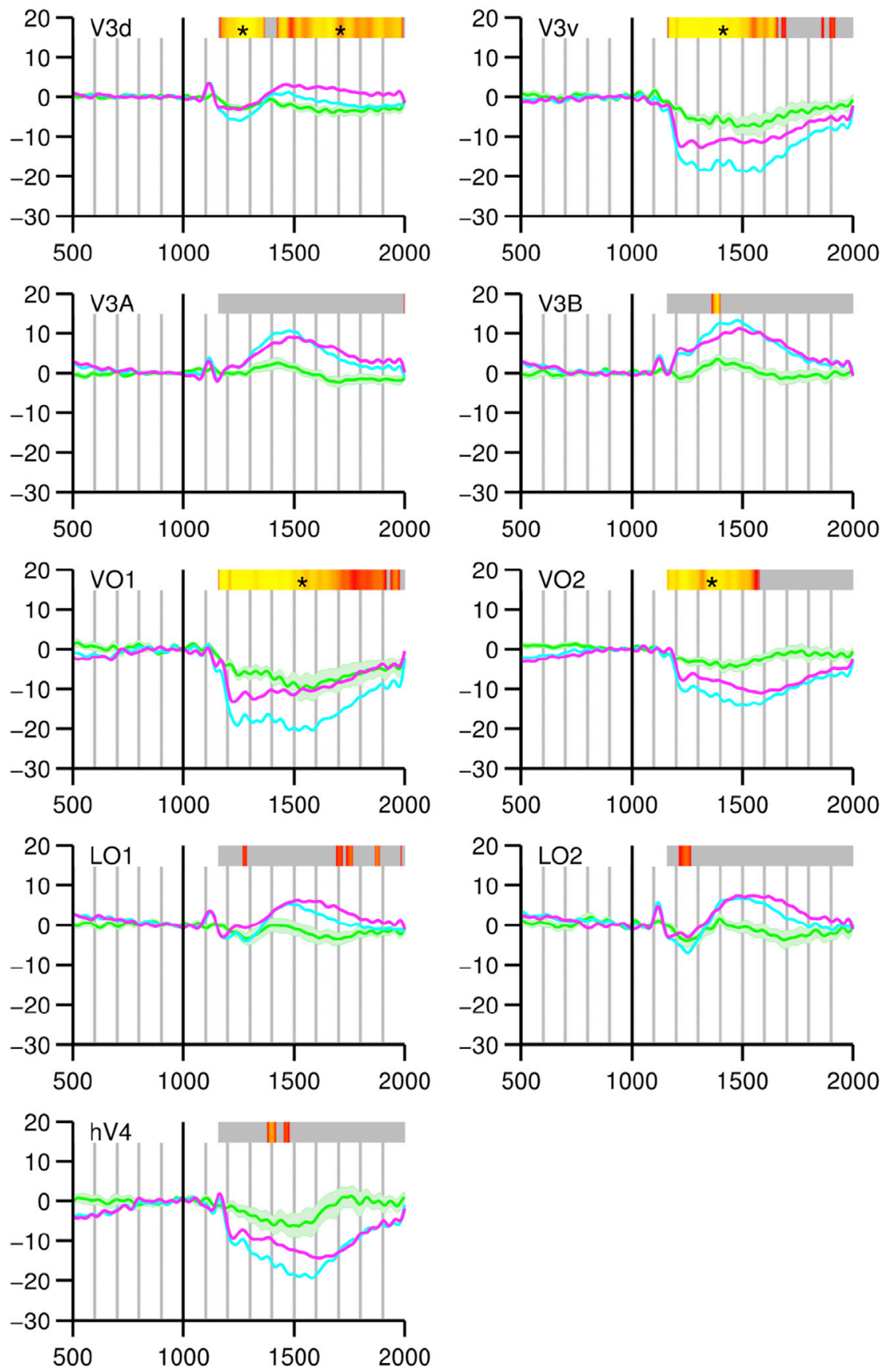

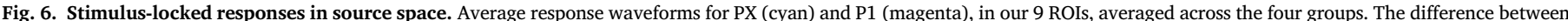

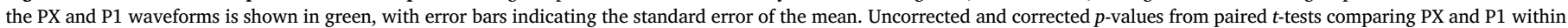

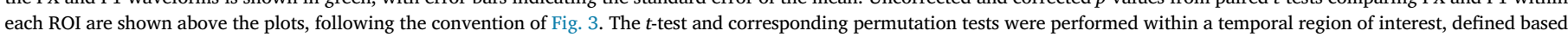

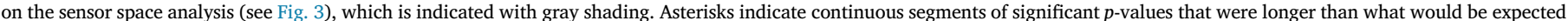
by chance, as indicated by permutation testing.

also had strong decision-related responses that began between 600 and $400 \mathrm{~ms}$ prior to participants' button presses (see Fig. 10). Our previous study also found that VO1 was one of three visual areas that had the strongest responses to rotation symmetry, along with hV4 and LOC (Kohler et al., 2016). Here we expand on this work by showing that VO1 is directly involved in perceptual decisions about symmetry. VO2, an area abutting VO1, also had both strong encoding-related and decision-related activity. VO1 and VO2 have received little attention compared to neighboring retinotopic areas hV4 and posterior parahippocampal cortex (PHC-1 and 2). The role of V4 in object perception and attention has been widely studied in both humans and monkey (Roe et al., 2012) and PHC-1 and 2 has substantial overlap with functionally localized parahippocampal place area (Arcaro et al., 2009). VO1 and VO2 have been mainly associated with color processing (Brewer et al., 2005; Brouwer and Heeger, 2009), which may interact with co-localized object representations (Arcaro et al., 2009; Vandenbroucke et al., 2014). The current results identify VO1 and VO2 as key players in symmetry processing, that not only exhibit strong encoding-related activity, but also directly drive perceptual decisions about symmetry.

\section{Precision of ROI-based source imaging measurements}

The precision of our source-imaging procedure deserves special consideration, given that the interpretation of our source-level analyses 

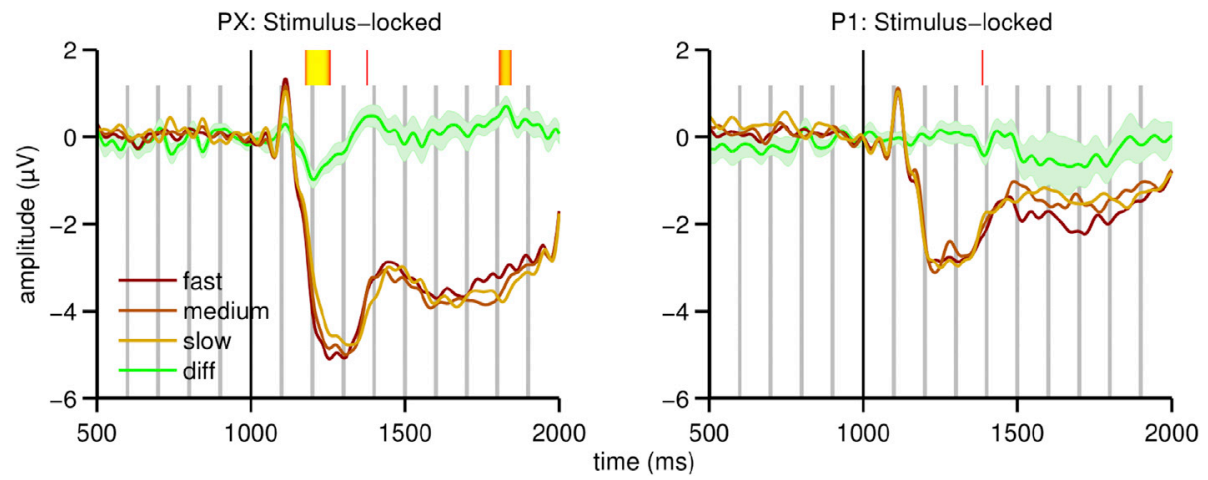

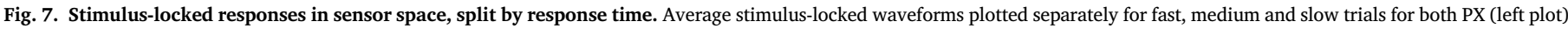

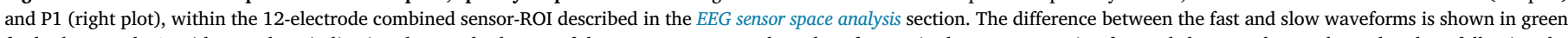

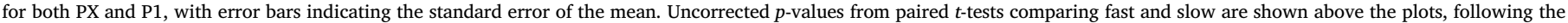
convention of Fig. 3. There were no continuous segments of significant $p$-values that permutation testing revealed as being longer than what would be expected by chance.
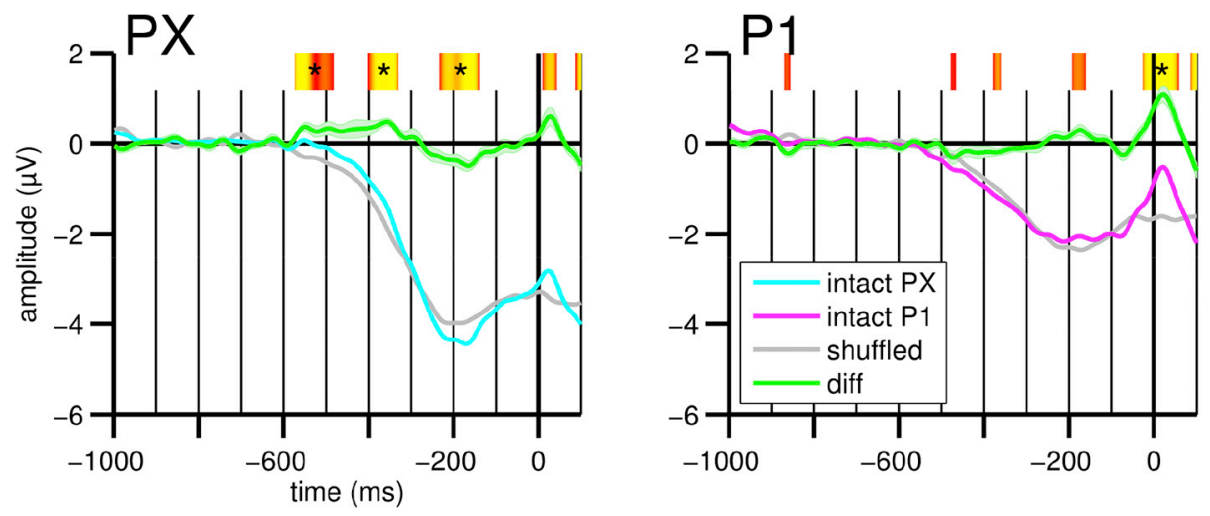

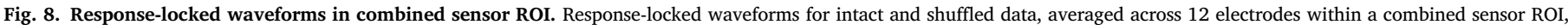

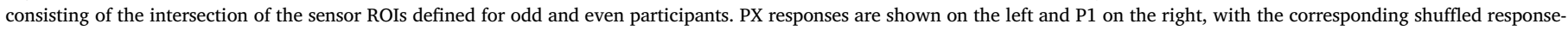

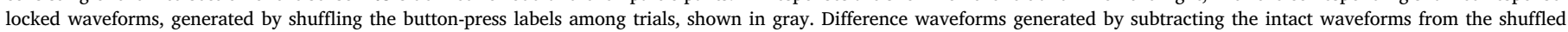

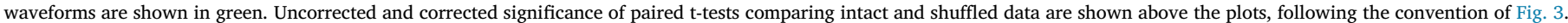

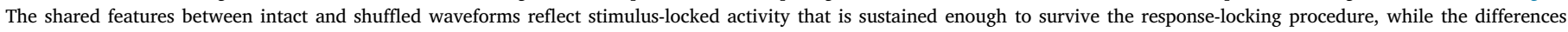
indicate activity that can be directly related to decision-making.

depends fundamentally on our ability to distinguish signals generated by different visual areas. Resolution in source-imaging depends not only on the spatial separation of sources, but also on the details of their 3D geometry. When a given set of spatially separated sources have different dominant orientations, they are easier to distinguish than sources with the same dominant orientation. Similarly, resolution also depends on source depth.

We have previously demonstrated that the ability of the minimum norm solution to distinguish activity is improved by averaging activity measured in individual ROIs across multiple participants, beyond the limits of the intrinsic resolution of the method in single participants (Cottereau et al., 2015). Because of the inherent properties of the EEG inverse problem and specific limitations of the L2 inverse, activity in a given ROI, hV4 for example, is a function not only of the activity arising from hV4, but also from activity in neighboring ROIs. We have shown in detailed simulations that the activity from neighboring ROIs will in some cases project with a positive sign into a given ROI, and in other cases project with a negative sign (Cottereau et al., 2015). This detailed pattern of cross-talk signs varies considerably across individuals. By averaging in the ROI space across participants, cross-talk between ROIs decreases as the number of participants in the average increases. The decrease is rapid up to approximately 8 participants, with a slower improvement for larger numbers (Cottereau et al., 2015). This property is a unique advantage of the way we perform ROI-based source imaging.
With this in mind, how confident can we be in the spatial precision of the source-imaging approach used in the present study? Barring the availability of independent, ground truth evidence, we can assess the resolution of our method by asking whether the source-waveforms or patterns of response over conditions are the same or different in different ROIs. ROI-level sources that have not been resolved will have the same waveform or response profile. ROI-sources that have been resolved may have different waveforms or response profiles depending on the functional specificity of the ROIs.

Multiple dissociations between ROI functional profiles are apparent in our data and these suggest that our method has an adequate level of resolution for the questions we are asking. Although hV4 responses were more pronounced for the PX than for the P1 condition (see Fig. 6), no runs of consecutively significant time-points survived our criterion for corrected significance, indicating that hV4 did not exhibit encodingrelated responses to symmetry. This null result would appear to contradict our previous study where we did observe significant symmetry responses in hV4 (Kohler et al., 2016). It is important to note, however, that in our present study hV4 had larger error bars than any other area (see Fig. 6). It is therefore plausible that hV4 did in fact exhibit encoding-related responses to symmetry, that our approach was not sensitive enough to detect. Importantly, areas V3A, V3B, LO1 and LO2 all showed no symmetry-specific stimulus-locked activity (see Fig. 6), indicating that those areas could be resolved from both the ventral areas, and 

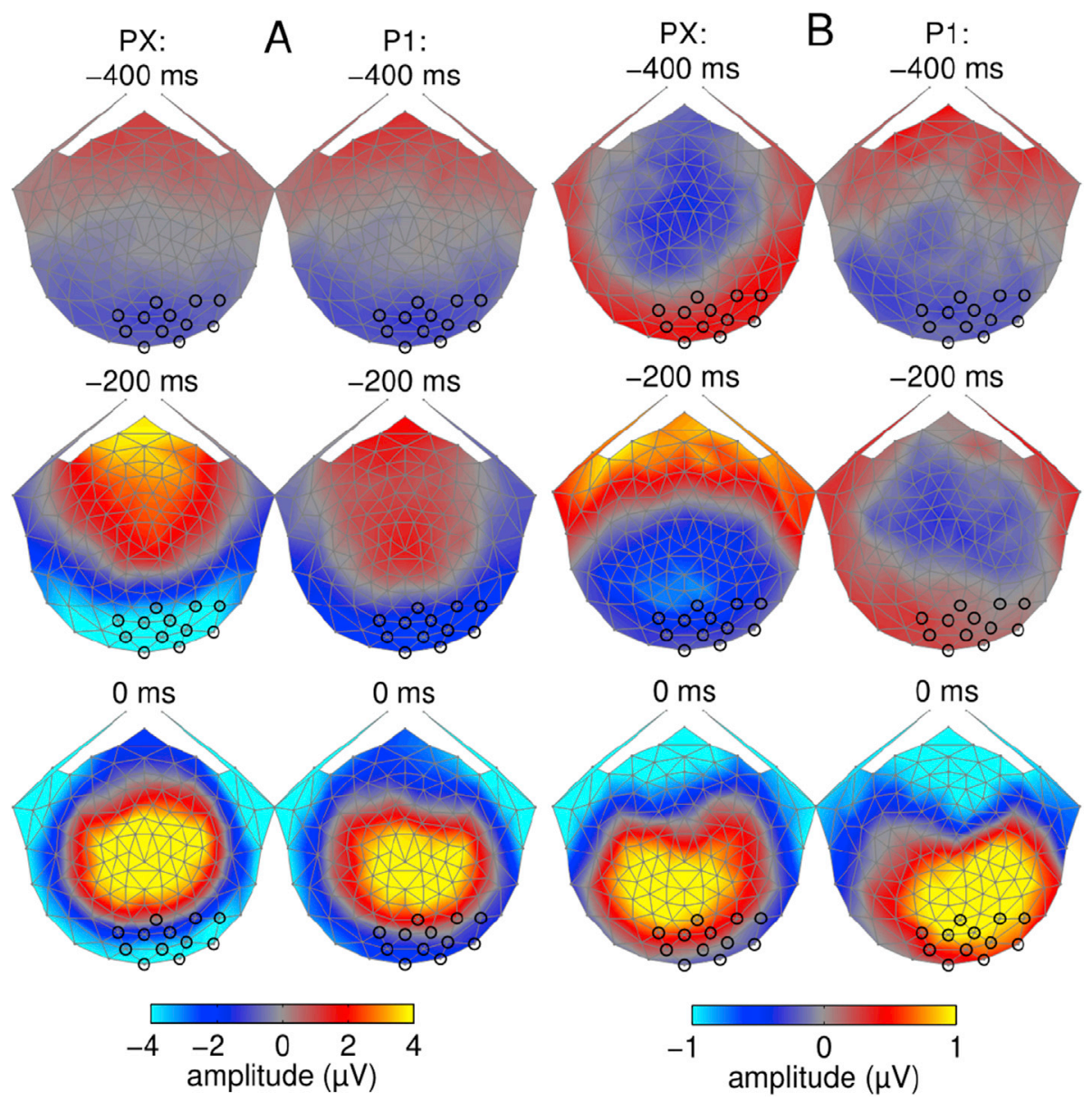

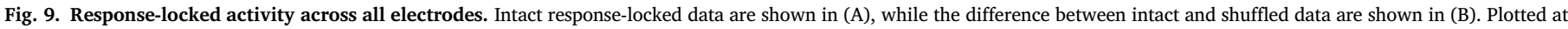
400 (top) and $200 \mathrm{~ms}$ (middle) prior to the button-press, and at the moment of the button press (bottom).

nearby dorsal V3, all of which had symmetry-specific responses (see Fig. 5).

From this one could argue that V3, hV4, VO1 and VO2 were essentially unresolved at the functional level. In the response-locked analysis, however, there were several dissociations between these areas. We found evidence of decision-related activity in ventral V3, hV4 and VO1, but not in dorsal V3 (see Fig. 10). Our previous study found that there was no functional distinction between dorsal and ventral V3 (Kohler et al., 2016), so the decision-related activity we see in ventral V3 is likely due to cross-talk from VO1 or hV4, rather than activity arising from ventral V3 itself. From these results, we conclude that dorsal, but not ventral V3, can be resolved from VO1.

The pattern just described suggests that the dominant locus of decision-related activity lies in hV4/VO1/VO2. A close analysis of the response timing suggests that these three areas have also been resolved: Consistent decision-related activity in hV4 and VO2 began $\sim 100$ ms later than the corresponding decision-related activity in VO1 (see Fig. 10). Taking both the stimulus- and response-locked data together, our results suggest that while hV4 and VO2 might have both stimulus- and decisiondriven activity, VO1 is the earliest and primary driver of perceptual decisions about symmetry.

\section{Relationship to prior work on the cortical locus of symmetry perception}

Previous functional MRI, TMS and EEG studies have implicated LOC in symmetry processing (Bona et al., 2014; Kohler et al., 2016; Sasaki et al., 2005; Tyler et al., 2005). LO1 and LO2, retinotopic areas that overlap with functionally defined LOC (Larsson and Heeger, 2006), did not have a measurable symmetry-specific response in the stimulus-locked analysis (see Fig. 6). The absence here of differential encoding of symmetry in LO1 and LO2 thus contrasts with results from the previous studies. We note, however, that most of these studies focused on reflection, rather than rotation symmetry (Bona et al., 2014; Sasaki et al., 2005; Tyler et al., 2005) and none of these studies used wallpaper patterns. These factors, either separately, or in combination could contribute to the differences in the overall pattern of symmetry-specific activity between the present study and previous work.

In our previous study of rotation symmetry, we did use wallpaper patterns and did find activation in LOC (Kohler et al., 2016). There are however several differences between the present study and our previous one. Here we studied symmetry processing in an open-loop fashion - up to the time of the second interval in our task, there was no prior information regarding stimulus category and the decision needed to be made based on a single pattern exemplar. In our previous study, symmetry patterns from a given group were presented periodically at $0.8 \mathrm{~Hz}$ over $12 \mathrm{sec}$ trials, allowing ample time for feedback activity to influence the response after the first presentation. It is possible that repeated presentation allowed for higher-level object processing in the LOC that is less likely to be evoked than in single, unpredictable event-related stimulus presentations. Previous work directly comparing reflection to rotation symmetry (Makin et al., 2012) has found differences between the two in terms of response waveform, time and source distribution, with the response to rotation symmetry producing a smaller Sustained Posterior Negativity (SPN) with a more diffuse source distribution. If rotation symmetry generates smaller responses than reflection symmetry, our current paradigm may be subject to a floor effect in LO1 and LO2. Finally, it is worth noting that LO2 (but not LO1) does exhibit consistent differences between the intact and shuffled response-locked waveforms at or 
$P X$

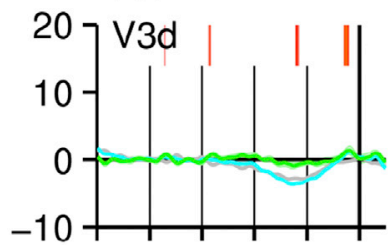

$-1000-600-2000$

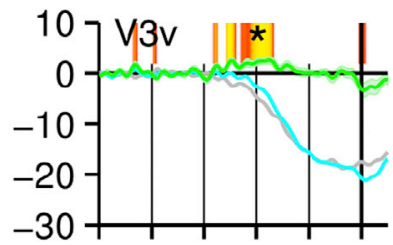

$-1000-600-2000$

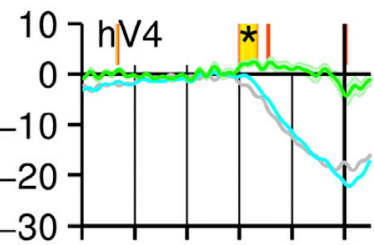

$-1000-600-2000$

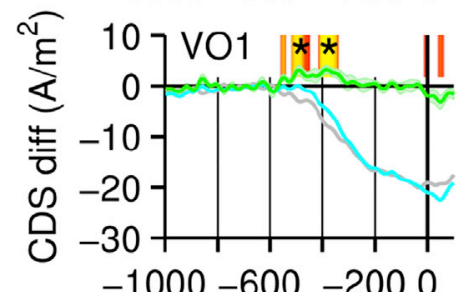

$-1000-600-2000$

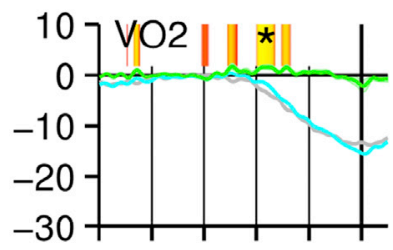

$-1000-600-2000$

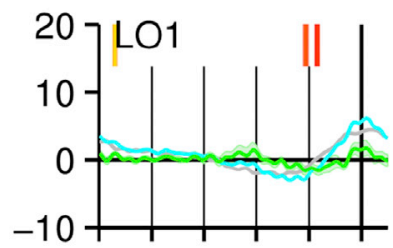

$-1000-600-2000$

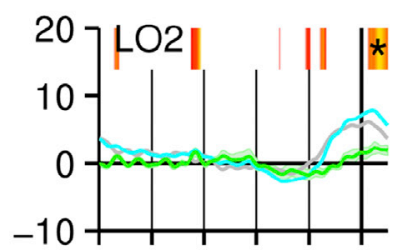

$-1000-600-2000$
P1

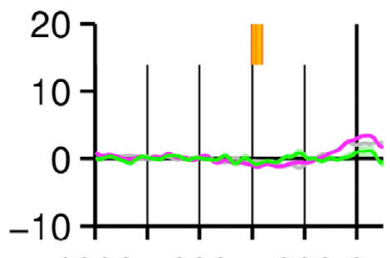

$-1000-600-2000$

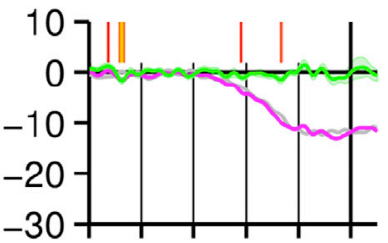

$-1000-600-2000$

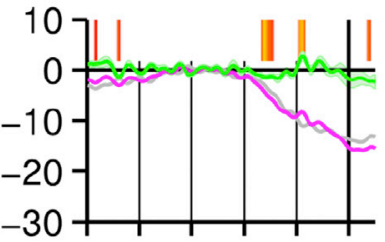

$-1000-600-2000$
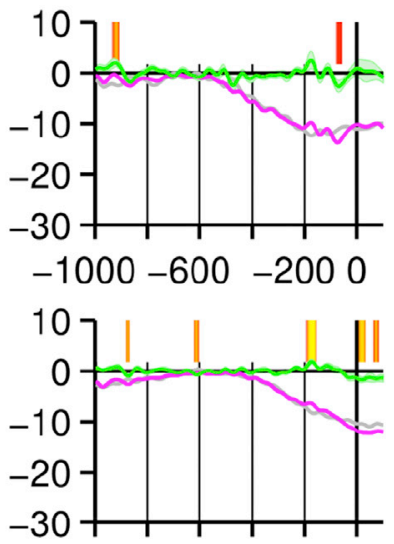

$-1000-600-2000$

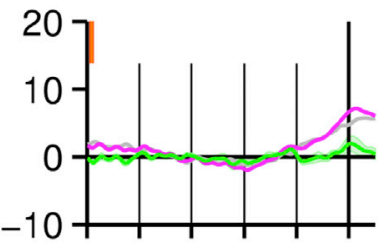

$-1000-600-2000$

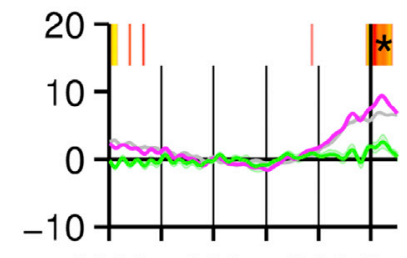

$-1000-600-2000$

time (ms)

Fig. 10. Responses-locked waveforms for intact data source-localized to 7 visual ROIs. Response-locked waveforms averaged across sources within seven ROIs, four of which showed strong symmetry responses in the stimulus-locked analysis (V3d, V3v, VO1 and VO2). The plots follow the convention from Fig. 8: PX responses are shown on the right and $\mathrm{P} 1$ on the left, with the corresponding shuffled response-locked waveforms, generated by shuffling the button-press labels among trials, shown in gray. Difference waveforms

immediately after the button-press. This suggests that LO2 plays a role after the decision has been made, potentially providing a confidence signal. Further experiments under both open- and closed loop conditions will be necessary to determine the role of LO1 and LO2 in symmetry processing.

\section{Relationship to prior work on the dynamics of symmetry perception}

Our sensor-space analysis of encoding-related activity revealed a symmetry-specific response in electrode ROIs over occipital cortex defined using an independent feature selection procedure. The amplitudes of the stimulus-locked activity varied with the maximum order of rotation symmetry present in each of the four wallpaper groups (see Supplementary Fig. S1), largely replicating our previous results (Kohler et al., 2016). Across all groups, there was a clear image-update response at $\sim 100 \mathrm{~ms}$ after the image onset, followed by a symmetry-specific response beginning at $\sim 150 \mathrm{~ms}$ and lasting until image offset (see Fig. 3). In our previous study (Kohler et al., 2016) we found evidence for symmetry-specific processing as early as $75 \mathrm{~ms}$. As discussed above, that study used a steady-state design, with repeated, predictable presentations, while the current event-related using single unpredictable stimuli. The higher predictability of the steady-state stimuli may have allowed for faster processing.

While the onset of the symmetry response at $150 \mathrm{~ms}$ may be later than what we previously observed, it is in fact earlier than the $\sim 220 \mathrm{~ms}$ previously seen for the SPN for reflection symmetry (Makin et al., 2013, 2012, 2016; Norcia et al., 2002). It is possible that the task demands in the current experiment, perhaps in combination with the use of rotation symmetry, create this difference in temporal dynamics, perhaps because different brain areas were recruited in our experiment. Support for different networks being involved in rotation and reflection symmetry comes from Makin et al. (2012) who found rotation symmetry-specific responses as early as $\sim 110 \mathrm{~ms}$, prior to the SPN generated by both rotation and reflection. These early responses were not observed for reflection. So both our current data and the previous study by Makin et al. (2012) find that neural responses to rotation may begin earlier than those for reflection. This is surprising given the psychophysical evidence that reflection symmetry can be detected more quickly than rotation (Palmer and Hemenway, 1978; Royer, 1981). In our data, the temporal progression of the scalp topography provides some evidence that different brain areas are recruited over time (see Fig. 4): The symmetry-specific activity begins medially and then quickly becomes more ventral and lateral localized (see Supplementary Video 1). Further exploration of the potential differences in the how the encoding of reflection and rotation symmetry unfolds over time, will be an important goal for future brain imaging work.

\section{Relation to previous work on perceptual decision making}

Our results add to a growing body of work which uses high-temporal resolution neuroimaging methods such as EEG to study perceptual decision-making in humans (Ales et al., 2013; Cottereau et al., 2014; Dmochowski and Norcia, 2015; Donner et al., 2009; Kelly and O'Connell, 2013; O'Connell et al., 2012; Philiastides et al., 2014). Traditional stimulus-locked analysis approaches identify cortical areas that contribute to the encoding of a given stimulus attribute. When considering whether an area might be causally involved in perceptual decisions about a stimulus attribute, evidence of encoding of this attribute is necessary, but not sufficient. Stimulus-locked analysis can identify

generated by subtracting the intact waveforms from the shuffled waveforms are shown in green. Uncorrected and corrected significance of paired t-tests comparing intact and shuffled data for each ROI are shown above the plots, following the convention of Fig. 3. There were consistent differences between intact and shuffled waveforms, prior to the button-press, indicating activity that can be directly related to decision-making, in V3d, V4, VO1 and VO2. 
encoding-related activity in a set of areas, but cannot determine which of them are causally involved in perceptual decisions. In previous work, we have shown that many early visual cortical areas encode disparity, but only a subset, hV4 in particular, are involved in decisions about depth magnitude (Cottereau et al., 2014). Similarly, many visual areas encode texture-defined form (Appelbaum et al., 2010, 2012, 2006), but only LOC is causally involved in decisions about shape (Ales et al., 2013). Our current results show that although $\mathrm{V} 3, \mathrm{hV} 4, \mathrm{VO} 1, \mathrm{VO} 2$ and lateral occipital cortex can all have stimulus-locked, encoding-related responses to rotation symmetry, only hV4, VO1 and VO2 can reliably be tied to perceptual decisions, with VO1 having the earliest, and therefore likely primary, decision-related variability. Based on this pattern across studies, we suggest a common computational strategy: encoding of perceptual attributes is widely distributed, but only a subset of the encoded information is read out for decision. The areas that primarily define this read-out appears to depend on the specific stimulus attribute in question: $\mathrm{hV} 4$ for disparity, LOC for form perception and VO1 for rotation symmetry.

\section{Conclusion}

In conclusion, our sensor space analyses identified encoding-related responses to rotation symmetry that began around $\sim 150 \mathrm{~ms}$ after the symmetry image was presented, somewhat earlier than what has been found in most previous studies of the latency of symmetry responses. Electrodes over occipital cortex, selected based on the strength of their encoding-related responses, also showed distinct decision-related activity that varied with button-press timing, beginning as early as $550 \mathrm{~ms}$ before participants pressed the button. Our source imaging analysis localized the encoding-related activity to several of the visual areas that have previously been found to respond to rotation symmetry, with the strongest symmetry-specificity occurring in area VO1. VO1 also exhibited robust decision-related activity that began earlier than any other area, indicating that VO1 may be the primary driver of perceptual decisions about rotation symmetry.

\section{Conflict-of-interest}

The authors declare no competing financial interests.

\section{Acknowledgements}

This research was supported by NSF grant INSPIRE 248076 awarded to AMN. The authors would like to thank Varun Bhadkamkar and David M. Altman for their contribution to the data collection for this study.

\section{Appendix A. Supplementary data}

Supplementary data related to this article can be found at https://doi. org/10.1016/j.neuroimage.2017.11.051.

\section{References}

Ales, J.M., Appelbaum, L.G., Cottereau, B.R., Norcia, A.M., 2013. The time course of shape discrimination in the human brain. Neuroimage 67, 77-88.

Appelbaum, L.G., Ales, J.M., Cottereau, B., Norcia, A.M., 2010. Configural specificity of the lateral occipital cortex. Neuropsychologia 48, 3323-3328.

Appelbaum, L.G., Ales, J.M., Norcia, A.M., 2012. The time course of segmentation and cue-selectivity in the human visual cortex. PLoS One 7, e34205.

Appelbaum, L.G., Wade, A.R., Vildavski, V.Y., Pettet, M.W., Norcia, A.M., 2006. Cueinvariant networks for figure and background processing in human visual cortex. J. Neurosci. 26, 11695-11708.

Apthorp, D., Bell, J., 2015. Symmetry is less than meets the eye. Curr. Biol. 25, R267-R268.

Arcaro, M.J., McMains, S.A., Singer, B.D., Kastner, S., 2009. Retinotopic organization of human ventral visual cortex. J. Neurosci. 29, 10638-10652.

Argall, B.D., Saad, Z.S., Beauchamp, M.S., 2006. Simplified intersubject averaging on the cortical surface using SUMA. Hum. Brain Mapp. 27, 14-27.

Baillet, S., Mosher, J.C., Leahy, R.M., 2001. Electromagnetic brain mapping. Signal processing magazine. IEEE 18, 14-30.
Bakeman, R., 2005. Recommended effect size statistics for repeated measures designs, Behav. Res. Methods 37, 379-384.

Bertamini, M., Makin, A., 2014. Brain activity in response to visual symmetry. Symmetry 6, 975-996.

Blair, R.C., Karniski, W., 1993. An alternative method for significance testing of waveform difference potentials. Psychophysiology 30, 518-524.

Bona, S., Herbert, A., Toneatto, C., Silvanto, J., Cattaneo, Z., 2014. The causal role of the lateral occipital complex in visual mirror symmetry detection and grouping: an fMRIguided TMS study. Cortex 51, 46-55.

Brewer, A.A., Liu, J., Wade, A.R., Wandell, B.A., 2005. Visual field maps and stimulus selectivity in human ventral occipital cortex. Nat. Neurosci. 8, 1102-1109.

Brouwer, G.J., Heeger, D.J., 2009. Decoding and reconstructing color from responses in human visual cortex. J. Neurosci. 29, 13992-14003.

Cattaneo, Z., Mattavelli, G., Papagno, C., Herbert, A., Silvanto, J., 2011. The role of the human extrastriate visual cortex in mirror symmetry discrimination: a TMSadaptation study. Brain Cognit. 77, 120-127.

Clarke, A.D.F., Green, P.R., Halley, F., Chantler, M.J., 2011. Similar symmetries: the role of wallpaper groups in perceptual texture similarity. Symmetry 3, 246-264.

Cohen, E.H., Zaidi, Q., 2013. Symmetry in context: salience of mirror symmetry in natural patterns. J. Vis. 13

Cottereau, B.R., Ales, J.M., Norcia, A.M., 2012. Increasing the accuracy of electromagnetic inverses using functional area source correlation constraints. Hum. Brain Mapp. 33, 2694-2713.

Cottereau, B.R., Ales, J.M., Norcia, A.M., 2014. The evolution of a disparity decision in human visual cortex. Neuroimage 92, 193-206.

Cottereau, B.R., Ales, J.M., Norcia, A.M., 2015. How to use fMRI functional localizers to improve EEG/MEG source estimation. J. Neurosci. Methods 250, 64-73.

Dakin, S., Watt, R., 1994. Detection of bilateral symmetry using spatial filters. Spat. Vis. 8, 393-413.

Dale, A.M., Fischl, B., Sereno, M.I., 1999. Cortical surface-based analysis: I. Segmentation and surface reconstruction. Neuroimage 9, 179-194.

Delius, J.D., Nowak, B., 1982. Visual symmetry recognition by pigeons. Psychol. Res. 44, 199-212.

DiCarlo, J.J., Maunsell, J.H.R., 2005. Using neuronal latency to determine sensory-motor processing pathways in reaction time tasks. J. Neurophysiol. 93, 2974-2986.

Dmochowski, J.P., Norcia, A.M., 2015. Cortical components of reaction-time during perceptual decisions in humans. PLoS One 10, e0143339.

Donner, T.H., Siegel, M., Fries, P., Engel, A.K., 2009. Buildup of choice-predictive activity in human motor cortex during perceptual decision making. Curr. Biol. 19, $1581-1585$.

Fedorov, E., 1891. Symmetry in the plane. Zapiski imperatorskogo S. Peterburgskogo mineralogichesgo obshchestva. Proc. S. Peterb. Mineral. Soc.

Gold, J.I., Shadlen, M.N., 2007. The neural basis of decision making. Annu. Rev. Neurosci. 30, 535-574.

Hadjikhani, N., Liu, A.K., Dale, A.M., Cavanagh, P., Tootell, R.B.H., 1998. Retinotopy and color sensitivity in human visual cortical area V8. Nat. Neurosci. 1, 235-241.

Hämäläinen, M., Hari, R., Ilmoniemi, R.J., Knuutila, J., Lounasmaa, O.V., 1993. Magnetoencephalography - theory, instrumentation, and applications to noninvasive studies of the working human brain. Rev. Mod. Phys. 65, 413.

Hämäläinen, M.S., Sarvas, J., 1989. Realistic conductivity geometry model of the human head for interpretation of neuromagnetic data. Biomedical Engineering. IEEE Trans. $36,165-171$.

Jacobsen, T., Höfel, L., 2003. Descriptive and evaluative judgment processes: behavioral and electrophysiological indices of processing symmetry and aesthetics. Cognitive, Affect. Behav. Neurosci. 3, 289-299.

Kelly, S.P., O'Connell, R.G., 2013. Internal and external influences on the rate of sensory evidence accumulation in the human brain. J. Neurosci. 33, 19434-19441.

Kohler, P.J., Clarke, A., Yakovleva, A., Liu, Y., Norcia, A.M., 2016. Representation of maximally regular textures in human visual cortex. J. Neurosci. 36, 714-729.

Kriegeskorte, N., Simmons, W.K., Bellgowan, P.S., Baker, C.I., 2009. Circular analysis in systems neuroscience: the dangers of double dipping. Nat. Neurosci. 12, 535-540.

Larsson, J., Heeger, D.J., 2006. Two retinotopic visual areas in human lateral occipital cortex. J. Neurosci. 26, 13128-13142.

Lawrence, M.A., 2015. ez: Easy Analysis and Visualization of Factorial Experiments.

Li, Y., Sawada, T., Shi, Y., Steinman, R., Pizlo, Z., 2013. Symmetry Is the sine qua non of Shape. In: Dickinson, S.J., Pizlo, Z. (Eds.), Shape Perception in Human and Computer Vision. Springer, London, pp. 21-40.

Liu, Y., Hel-Or, H., Kaplan, C.S., Van Gool, L., 2010. Computational symmetry in computer vision and computer graphics. Found. Trends ${ }^{\mathbb{B}}$ Comput. Graph. Vis. 5, $1-195$.

Lorenceau, J., Shiffrar, M., 1992. The influence of terminators on motion integration across space. Vis. Res. 32, 263-273.

Makin, A.D.J., Rampone, G., Bertamini, M., 2014. Conditions for view invariance in the neural response to visual symmetry. Psychophysiology 52, 532-543.

Makin, A.D., Rampone, G., Pecchinenda, A., Bertamini, M., 2013. Electrophysiological responses to visuospatial regularity. Psychophysiology 50, 1045-1055.

Makin, A.D., Wilton, M.M., Pecchinenda, A., Bertamini, M., 2012. Symmetry perception and affective responses: a combined EEG/EMG study. Neuropsychologia 50, 3250-3261.

Makin, A.D.J., Wright, D., Rampone, G., Palumbo, L., Guest, M., Sheehan, R., Cleaver, H., Bertamini, M., 2016. An Electrophysiological Index of Perceptual Goodness. Cereb Cortex 26, 4416-4434.

McDermott, J., Adelson, E.H., 2004. Motion perception and mid-level vision. In: Gazzaniga, M.S. (Ed.). The Cognitive Neurosciences III. MIT Press, Boston, pp. 369-383. 
Miller, J., Patterson, T., Ulrich, R., 1998. Jackknife-based method for measuring LRP onset latency differences. Psychophysiology 35, 99-115.

Miller, J., Ulrich, R., Schwarz, W., 2009. Why jackknifing yields good latency estimates. Psychophysiology 46, 300-312.

Møller, A.P., 1992. Female swallow preference for symmetrical male sexual ornaments. Nature 357, 238-240.

Mumford, D., Desolneux, A., 2010. Pattern Theory: the Stochastic Analysis of Real-world Signals. CRC Press.

Norcia, A.M., Candy, T.R., Pettet, M.W., Vildavski, V.Y., Tyler, C.W., 2002. Temporal dynamics of the human response to symmetry. J. Vis. 2.

O'Connell, R.G., Dockree, P.M., Kelly, S.P., 2012. A supramodal accumulation-to-bound signal that determines perceptual decisions in humans. Nat. Neurosci. 15, $1729-1735$.

Osman, A., Bashore, T.R., Coles, M.G.H., Donchin, E., Meyer, D.E., 1992. On the transmission of partial information: inferences from movement-related brain potentials. J. Exp. Psychol. Hum. Percept. Perform. 18, 217-232.

Palmer, S.E., Hemenway, K., 1978. Orientation and symmetry: effects of multiple rotational, and near symmetries. J. Exp. Psychol. Hum. Percept. Perform. 4, 691-702.

Palumbo, L., Bertamini, M., Makin, A., 2015. Scaling of the extrastriate neural response to symmetry. Vis. Res. 117, 1-8.

Pascual-Marqui, R.D., Michel, C.M., Lehmann, D., 1994. Low resolution electromagnetic tomography: a new method for localizing electrical activity in the brain. Int. J. Psychophysiol. 18, 49-65.

Philiastides, M.G., Heekeren, H.R., Sajda, P., 2014. Human scalp potentials reflect a mixture of decision-related signals during perceptual choices. J. Neurosci. 34, 16877-16889.
Polya, G., 1924. XII. Über die Analogie der Kristallsymmetrie in der Ebene. Z. für Kristallogr. Mater. 60, 278-282.

R Core Team, 2014. R: a Language and Environment for Statistical Computing (Vienna, Austria).

Roe, A.W., Chelazzi, L., Connor, C.E., Conway, B.R., Fujita, I., Gallant, J.L., Lu, H., Vanduffel, W., 2012. Toward a unified theory of visual area V4. Neuron 74, 12-29.

Royer, F.L., 1981. Detection of symmetry. J. Exp. Psychol. Hum. Percept. Perform. 7 , 1186-1210.

Sasaki, Y., Vanduffel, W., Knutsen, T., Tyler, C., Tootell, R., 2005. Symmetry activates extrastriate visual cortex in human and nonhuman primates. Proc. Natl. Acad. Sci. U. S. A. 102, 3159-3163.

Smith, S.M., 2002. Fast robust automated brain extraction. Hum. Brain Mapp. 17, 143-155.

Smith, S.M., Jenkinson, M., Woolrich, M.W., Beckmann, C.F., Behrens, T.E., JohansenBerg, H., Bannister, P.R., De Luca, M., Drobnjak, I., Flitney, D.E., 2004. Advances in functional and structural MR image analysis and implementation as FSL. Neuroimage 23, S208-S219.

Swisher, J.D., Halko, M.A., Merabet, L.B., McMains, S.A., Somers, D.C., 2007. Visual topography of human intraparietal sulcus. J. Neurosci. 27, 5326-5337.

Tyler, C.W., Baseler, H.A., Kontsevich, L.L., Likova, L.T., Wade, A.R., Wandell, B.A., 2005. Predominantly extra-retinotopic cortical response to pattern symmetry. Neuroimage 24, 306-314.

Vandenbroucke, A.R.E., Fahrenfort, J.J., Meuwese, J.D.I., Scholte, H.S., Lamme, V.A.F., 2014. Prior Knowledge about Objects Determines Neural Color Representation in Human Visual Cortex. Cereb. Cortex 26, 1401-1408.

Wang, L., Mruczek, R.E.B., Arcaro, M.J., Kastner, S., 2015. Probabilistic Maps of Visual Topography in Human Cortex. Cereb. Cortex 25, 3911-3931. 